\title{
On exponential functionals of Lévy processes
}

\author{
Anita Behme* and Alexander Lindner ${ }^{\dagger}$
}

August 18, 2018

\begin{abstract}
Exponential functionals of Lévy processes appear as stationary distributions of generalized Ornstein-Uhlenbeck (GOU) processes. In this paper we obtain the infinitesimal generator of the GOU process and show that it is a Feller process. Further we use these results to investigate properties of the mapping $\Phi$, which maps two independent Lévy processes to their corresponding exponential functional, where one of the processes is assumed to be fixed. We show that in many cases this mapping is injective and give the inverse mapping in terms of (Lévy) characteristics. Also, continuity of $\Phi$ is treated and some results on its range are obtained.
\end{abstract}

2000 Mathematics subject classification. 60G10, 60G51, 60J35.

Key words and phrases. generalized Ornstein-Uhlenbeck process, Lévy process, Feller process, infinitesimal generator, integral mapping, stationarity

\section{Introduction}

The exponential functional of a bivariate Lévy process $(\xi, \eta)^{T}=\left(\left(\xi_{t}, \eta_{t}\right)^{T}\right)_{t \geq 0}$ is defined as

$$
V_{\infty}=\int_{(0, \infty)} e^{-\xi_{t-}} d \eta_{t}
$$

Necessary and sufficient conditions for the convergence of integrals of the form $\int_{(0, t]} e^{-\xi_{s-}} d \eta_{s}$ as $t \rightarrow \infty$ for a bivariate Lévy process $(\xi, \eta)^{T}$ were given by Erickson and Maller [12, Thm. $2]$. Distributional properties of exponential functionals have been studied in various articles throughout the years by e.g. Paulsen [34], Yor [41], Bertoin et al. [6], Kondo et al. [22], Lindner and Sato [28], Behme [3] and Kuznetsov et al. [23] to name just a few.

\footnotetext{
*Technische Universität München, Institut für Mathematische Statistik, Boltzmannstraße 3, D-85748 Garching bei München, Germany, email: a.behme@tum.de, tel.: +49/89/28917424, fax:+49/89/28917435

${ }^{\dagger}$ Technische Universität Braunschweig, Institut für Mathematische Stochastik, Pockelsstr. 14, D-38106 Braunschweig, Germany, email: a.lindner@tu-bs.de, tel.:+49/531/3917575, fax:+49/531/3917564
} 
Denote by $\mathcal{L}(X)$ the law of a random variable $X$. In this paper, for a given one-dimensional Lévy process $\xi$, we will consider mappings like

$$
\begin{aligned}
\Phi_{\xi}: D_{\xi} & \rightarrow \text { set of probability distributions on } \mathbb{R} \\
\mathcal{L}\left(\eta_{1}\right) & \mapsto \mathcal{L}\left(\int_{0}^{\infty} e^{-\xi_{s-}} d \eta_{s}\right)
\end{aligned}
$$

defined on $D_{\xi}:=\left\{\mathcal{L}\left(\eta_{1}\right): \eta\right.$ Lévy process, independent of $\xi$, such that $\int_{0}^{\infty} e^{-\xi_{s-}} d \eta_{s}$ converges a.s. $\}$ and we will examine injectivity and continuity of such mappings and gather information about their ranges. In the case that $\xi_{t}=a t$ is deterministic, it is well known that $D_{\xi}=\mathrm{ID}_{\log }(\mathbb{R})$ is the set of real-valued infinitely divisible distributions with finite $\log ^{+}$-moment and that $\Phi_{\xi}$ is an algebraic isomorphism between $\operatorname{ID}_{\log }(\mathbb{R})$ and $L(\mathbb{R})$, the set of real-valued selfdecomposable distributions [16, Prop. 3.6.10].

We start with a short example of a special case to illustrate the kind of results we obtain, as well as the occuring problems.

Example 1.1. Suppose $\left(\xi_{t}\right)_{t \geq 0}$ is a compound Poisson process with intensity rate $\lambda$ and jump heights measure $\tau$. Let $\eta$ be a Lévy process independent of $\xi$ such that $\mathcal{L}\left(\eta_{1}\right) \in D_{\xi}$. Define $T_{i}$ to be the time of the ith jump of $\xi$ with $T_{0}:=0$. Then

$$
V_{\infty}=\int_{(0, \infty)} e^{-\xi_{s-}} d \eta_{s}=\sum_{i=0}^{\infty} \int_{\left(T_{i}, T_{i+1}\right]} e^{-\xi_{T_{i}}} d \eta_{t}=\sum_{i=0}^{\infty}\left(\prod_{k=1}^{i} e^{-\Delta \xi_{T_{k}}}\right)\left(\eta_{T_{i+1}}-\eta_{T_{i}}\right)
$$

Since $\left(e^{-\Delta \xi_{T_{i}}}, \eta_{T_{i+1}}-\eta_{T_{i}}\right)_{i=0,1,2, \ldots}$ is an i.i.d. sequence, as e.g. in [5] we obtain from this the distributional fixed point equation

$$
V_{\infty} \stackrel{d}{=} X V_{\infty}^{\prime}+H
$$

where $(X, H) \stackrel{d}{=}\left(e^{-\Delta \xi_{T_{i}}}, \eta_{T_{i+1}}-\eta_{T_{i}}\right)$ for $i=1,2, \ldots$ and $V_{\infty} \stackrel{d}{=} V_{\infty}^{\prime}$ where $V_{\infty}^{\prime}$ is independent of $(X, H)$. In terms of characteristic functions this yields $\phi_{V_{\infty}}(u)=\phi_{X V_{\infty}^{\prime}}(u) \phi_{H}(u)$ and adding the fact that the characteristic function $\phi_{\eta}$ of the Lévy process $\left(\eta_{t}\right)_{t \geq 0}$ and the corresponding exponentially subordinated process $\left(H_{t}\right)_{t \geq 0}=\left(\eta_{\tau(t)}\right)_{t \geq 0}$ with $\tau \sim \operatorname{Exp}(\lambda)$ fulfill the equation

$$
\phi_{H}(u)=\frac{\lambda}{\lambda-\log \left(\phi_{\eta}(u)\right)}
$$

(see e.g. 40, p.10]) we have

$$
\log \left(\phi_{\eta}(u)\right) \phi_{V_{\infty}}(u)=\lambda\left(\phi_{V_{\infty}}(u)-\phi_{X V_{\infty}^{\prime}}(u)\right)=\lambda \int_{\mathbb{R}}\left(E\left[e^{i u V_{\infty}}\right]-E\left[e^{i u e^{-y} V_{\infty}}\right]\right) \tau(d y) .
$$

Now, in the setting of the example if we knew that the characteristic function of $V_{\infty}$ is non-zero on a dense subset of $\mathbb{R}$ this gave us a formula for the characteristic exponent of $\eta$ and thus injectivity of the mapping $\Phi_{\xi}$. But in general the quotient of two characteristic functions does not necessarily yield a unique solution as has already been remarked in [29]. Examples for non-uniqueness of such quotients are also given in [25]. 
To obtain formulas like (1.2) for general Lévy processes $(\xi, \eta)^{T}$ we will strongly make use of the fact that GOU processes are Markov processes. So, in Section 3 we first compute the infinitesimal generator of the GOU process and show that it is actually a Feller process. In Section 4 these results will be used to obtain formulas of the form (1.2) for general, independent Lévy processes $\xi$ and $\eta$. Hereby we obtain a general formula for $\log \left(\phi_{\eta}(u)\right) \phi_{V_{\infty}}(u)$ in terms of the characteristic triplet of $\xi$ and $\mathcal{L}\left(V_{\infty}\right)$ as given in Theorem 4.1 and Corollary 4.3 and on the other hand in Theorem 4.6 we express $\log \left(\phi_{-\xi}(u)\right) \phi_{\log \left|V_{\infty}\right|}(u)$ in terms of the characteristic triplet of $\eta$ and $\mathcal{L}\left(V_{\infty}\right)$.

Further, Section 5 is devoted to the study of injectivity, which - in view of the results of Section 4 - now reduces to an examination of when either $\phi_{V_{\infty}}(u)$ or $\phi_{\log \left|V_{\infty}\right|}(u)$ are non-zero on a dense subset of $\mathbb{R}$. We give various examples of when the mapping $\Phi_{\xi}$ or its counterpart $\tilde{\Phi}_{\eta}$ (which maps $\mathcal{L}\left(\xi_{1}\right)$ to $\mathcal{L}\left(V_{\infty}\right)$ for $\eta$ fixed) are injective and argue why injectivity cannot be obtained if $\xi$ and $\eta$ are allowed to exhibit a dependence structure.

Section 6 then uses the previous results to obtain information on the ranges of $\Phi_{\xi}$ and $\tilde{\Phi}_{\eta}$. In particular, Theorem 6.4 shows that centered Gaussian distributions can only be obtained in the setting of (standard) OU processes, i.e. for $\xi$ being deterministic and $\eta$ being a Brownian motion.

Finally, in Section 7 we give conditions for continuity (in a weak sense) of the mappings $\Phi_{\xi}$ and $\tilde{\Phi}_{\eta}$ and give an example of $\Phi_{\xi}$ being not continuous.

\section{Some background on GOU processes and Nota- tions}

By the Lévy-Khintchine formula (e.g. [36, Thm. 8.1]) the characteristic exponent of an $\mathbb{R}^{d}$-valued Lévy process $X=\left(X_{t}\right)_{t \geq 0}$ is given by

$$
\begin{aligned}
\psi_{X}(u) & :=\log \phi_{X}(u):=\log E\left[e^{i\left\langle u, X_{1}\right\rangle}\right] \\
& =i\left\langle\gamma_{X}, u\right\rangle-\frac{1}{2}\left\langle u, A_{X} u\right\rangle+\int_{\mathbb{R}^{d}}\left(e^{i\langle u, x\rangle}-1-i\langle u, x\rangle \mathbb{1}_{|x| \leq 1}\right) \nu_{X}(d x)
\end{aligned}
$$

where $\left(\gamma_{X}, A_{X}, \nu_{X}\right)$ is the characteristic triplet of $X$. In case that $X$ is real valued we will usually replace $A_{X}$ by $\sigma_{X}^{2}$. To simplify notations, we set $\nu(\{0\})=0$ for any Lévy measure $\nu$. If the Lévy measure $\nu_{X}$ satisfies the condition $\int_{|x| \leq 1}|x| \nu_{X}(d x)<\infty$ we may also use the Lévy-Khintchine formula in the form

$$
\psi_{X}(u)=i\left\langle\gamma_{X}^{0}, u\right\rangle-\frac{1}{2}\left\langle u, A_{X} u\right\rangle+\int_{\mathbb{R}^{d}}\left(e^{i\langle u, x\rangle}-1\right) \nu_{X}(d x)
$$

and call $\gamma_{X}^{0}$ the drift of $X$. We refer to [36] for any further information on Lévy processes. We write $\Delta Y_{t}=Y_{t}-Y_{t-}$ for any càdlàg process $Y$.

Given a bivariate Lévy process $\left(\left(\xi_{t}, \eta_{t}\right)^{T}\right)_{t \geq 0}$ and a random variable $V_{0}$ on the same probability space,

$$
V_{t}=e^{-\xi_{t}}\left(\int_{0}^{t} e^{\xi_{s-}} d \eta_{s}+V_{0}\right), \quad t \geq 0
$$


defines the generalized Ornstein-Uhlenbeck (GOU) process driven by $(\xi, \eta)^{T}$ with starting random variable $V_{0}$. In the case that $\xi_{t}=a t$ is deterministic, the process $V_{t}$ is usually called Ornstein-Uhlenbeck-type process, while if $\left(\xi_{t}, \eta_{t}\right)=\left(a t, B_{t}\right)$ for $B$ a Brownian motion, $V_{t}$ is known as Ornstein-Uhlenbeck (OU) process.

The GOU process driven by $(\xi, \eta)^{T}$ is the unique solution of the stochastic differential equation

$$
d V_{t}=V_{t-} d U_{t}+d L_{t}, \quad t \geq 0
$$

for the bivariate Lévy process $\left(\left(U_{t}, L_{t}\right)^{T}\right)_{t \geq 0}$ given by

$$
\left(\begin{array}{c}
U_{t} \\
L_{t}
\end{array}\right)=\left(\begin{array}{l}
-\xi_{t}+\sum_{0<s \leq t}\left(e^{-\Delta \xi_{s}}-1+\Delta \xi_{s}\right)+t \sigma_{\xi}^{2} / 2 \\
\eta_{t}+\sum_{0<s \leq t}\left(e^{-\Delta \xi_{s}}-1\right) \Delta \eta_{s}-t \sigma_{\xi, \eta}
\end{array}\right), \quad t \geq 0,
$$

where $\sigma_{\xi}^{2}$ and $\sigma_{\xi, \eta}$ denote the $(1,1)$ and $(1,2)$ elements of the Gaussian covariance matrix $A_{(\xi, \eta)}$. Equation (2.3) defines a bijection between all bivariate Lévy processes $(\xi, \eta)^{T}$ and all bivariate Lévy process $(U, L)^{T}$ such that $\nu_{U}((-\infty,-1])=0$. The upper line of $(2.3)$ is equivalent to $e^{-\xi_{t}}=\mathcal{E}(U)_{t}$, where $\mathcal{E}(U)_{t}$ is the stochastic exponential of $U$, which is defined as the unique càdlàg solution $S$ of $S_{t}=1+\int_{(0, t]} S_{s-} d U_{s}$ (see e.g. [35, Thm. II.37]). Equation (2.2) has a solution for any bivariate Lévy process $(U, L)^{T}$ and any starting random variable $V_{0}$ independent of $(U, L)^{T}$, which in the case $\nu_{U}(\{-1\})=0$ is given by

$$
V_{t}=\mathcal{E}(U)_{t}\left(\int_{(0, t]} \mathcal{E}(U)_{s-}^{-1} d \eta_{s}+V_{0}\right)
$$

where $\eta_{t}=L_{t}-\sum_{0<s \leq t}\left(1+\Delta U_{s}\right)^{-1} \Delta U_{s} \Delta L_{s}-t \sigma_{U, L}$, see [4, Thm. 2.1]. The processes of the form (2.4) hence constitute a slightly larger class of stochastic processes than the GOU processes defined by (2.1) since they allow $U$ also to have jumps smaller than -1 . Obviously, the GOU process defined in (2.1) as well as the process defined in (2.4) are time homogeneous Markov processes [4, Lem. 3.3].

In [27] necessary and sufficient conditions for the existence of causal, strictly stationary solutions of the generalized Ornstein-Uhlenbeck process (2.1) are given. In particular it is shown ([27, Thm. 2.1]) that if $\left(V_{t}\right)_{t \geq 0}$ is strictly stationary and causal, then $\int_{(0, t]} e^{-\xi_{s-}} d L_{s}$ with $L$ as defined in (2.3) converges a.s. to a finite random variable as $t \rightarrow \infty$ and the stationary law $\mu$ is given by $\mu=\mathcal{L}\left(V_{\infty}\right)$ for $V_{\infty}=\int_{(0, \infty)} e^{-\xi_{s-}} d L_{s}$. Observe that $L=\eta$ if $\xi$ and $\eta$ are independent.

The space of continuous functions $\mathbb{R}^{d} \rightarrow \mathbb{R}$ is denoted by $C\left(\mathbb{R}^{d}\right)$. The subspaces of bounded functions, functions vanishing at infinity and functions with compact support are written as $C_{b}\left(\mathbb{R}^{d}\right), C_{0}\left(\mathbb{R}^{d}\right)$ and $C_{c}\left(\mathbb{R}^{d}\right)$, resp. For $n \in \mathbb{N}$ we write $C^{n}\left(\mathbb{R}^{d}\right)$ for the space of functions which are $n$-times continuously differentiable. Functions in $C_{b}^{n}\left(\mathbb{R}^{d}\right)$ are $n$-times continuously differentiable and the first $n$ derivatives are bounded. $C_{0}^{n}\left(\mathbb{R}^{d}\right)$ and $C_{c}^{n}\left(\mathbb{R}^{d}\right)$ are defined likewise. For any bounded function $f$ we let $\|f\|=\|f\|_{\infty}$ denote its supremum norm. We write " $\stackrel{d}{=}$ to denote equality in distribution of random variables, $\stackrel{d}{\rightarrow}$ " to denote convergence in distribution of random variables, i.i.d. for "independent and identically distributed", and $\log ^{+}(x)=\log (\max \{x, 1\})$ for $x \in \mathbb{R}$. Throughout, the characteristic function of a random variable $X$ is denoted by $\phi_{X}(u)=E e^{i u X}, u \in \mathbb{R}$, and 
the Fourier transform of a finite measure $\mu$ on $\left(\mathbb{R}, \mathcal{B}_{1}\right)$ by $\widehat{\mu}(u)=\int_{\mathbb{R}} e^{i u x} \mu(d x)$. Here, $\mathcal{B}_{1}$ denotes the Borel- $\sigma$-algebra in $\mathbb{R}$.

\section{Feller property and the infinitesimal generator of the GOU process}

Let $\left(X_{t}\right)_{t \geq 0}$ be a time homogeneous Markov process on $\mathbb{R}^{d}$ with semigroup $T_{t}$, i.e.

$$
T_{t} f(x)=\int_{\mathbb{R}^{d}} f(y) \mu_{t}(x, d y)=E^{x}\left[f\left(X_{t}\right)\right]
$$

where $\mu_{t}(x, d y)=P\left(X_{t} \in d y \mid X_{0}=x\right)$ are the transition probabilities of $X$ and $f \in$ $C_{0}\left(\mathbb{R}^{d}\right)$. Then $X$ is a Feller process in $\mathbb{R}^{d}$ if its semigroup fulfills the Feller properties

(F1) $\quad T_{t} C_{0}\left(\mathbb{R}^{d}\right) \subset C_{0}\left(\mathbb{R}^{d}\right)$

(F2) $\quad T_{t} f \rightarrow f$ as $t \rightarrow 0 \quad \forall f \in C_{0}\left(\mathbb{R}^{d}\right)$,

where the convergence under (F2) is meant to hold in the Banach space $\left(C_{0}\left(\mathbb{R}^{d}\right),\|\cdot\|_{\infty}\right)$. The infinitesimal generator $A^{X}$ of a Feller process $X$ is defined by

$$
A^{X} f=\lim _{t \rightarrow 0} \frac{T_{t} f-f}{t}
$$

for all functions $f$ in the domain of $A^{X}$, i.e. all $f$ in

$$
D\left(A^{X}\right)=\left\{f \in C_{0}\left(\mathbb{R}^{d}\right), \lim _{t \rightarrow 0} \frac{T_{t} f-f}{t} \text { exists in }\|\cdot\|_{\infty}\right\} .
$$

A subspace $D$ of $D\left(A^{X}\right)$ is said to be a core for the generator $A^{X}$, if the closure of the restriction of $A^{X}$ to $D$ is equal to $A^{X}$.

Every Lévy process $X$ is a Feller process. If the Lévy process $X$ is real-valued its generator $A^{X}$ is given by (e.g. [36, Thm. 31.5])

$$
A^{X} f(x)=\frac{1}{2} \sigma_{X}^{2} f^{\prime \prime}(x)+\gamma_{X} f^{\prime}(x)+\int_{\mathbb{R}}\left(f(x+y)-f(x)-y f^{\prime}(x) \mathbb{1}_{|y| \leq 1}\right) \nu_{X}(d y)
$$

and it holds $C_{0}^{2}(\mathbb{R}) \subset D\left(A^{X}\right)$.

The generator of the OU process is well known in the literature, unlike the generator of the GOU process, which is presented in the next theorem. For Lévy processes with finite second moment this generator is also given in [21, Thm. 4.6.1] and the formula for the generator can also be found in [35, Exercise V.7] (containing a typo). The fact that GOU processes are Feller processes and the determination of the cores seems to be new.

Note that the equation $d V_{t}^{x}=x+\int_{(0, t]} V_{s-}^{x} d U_{s}+d L_{t}$ can be written as

$$
d V_{t}^{x}=x+\int_{(0, t]} g\left(V_{s-}^{x}\right) d\left(U_{s}, L_{s}\right)^{T}
$$


with $g(u)=(u, 1) \in \mathbb{R}^{1 \times 2}$. Solutions of (3.2) with bounded and locally Lipschitz $g$ are well known to constitute Feller process (e.g. [39, Cor. 3.3]), but the function $u \mapsto(u, 1)$ is not bounded so that this theory cannot be applied. Further, in [39, Rem. 3.4] an example is given when $g$ is not bounded and the corresponding solution fails to be a Feller process.

Theorem 3.1. Let $\left(Z_{t}\right)_{t \geq 0}=\left(\left(U_{t}, L_{t}\right)^{T}\right)_{t \geq 0}$ be a bivariate Lévy process with characteristic triplet $\left(\gamma_{Z}, A_{Z}, \nu_{Z}\right)$ where $\gamma_{Z}=\left(\gamma_{U}, \gamma_{L}\right)^{T}, A_{Z}=\left(\begin{array}{cc}\sigma_{U}^{2} & \sigma_{U, L} \\ \sigma_{U, L} & \sigma_{L}^{2}\end{array}\right)$ and $\nu_{Z}\left(\left(d z_{1}, d z_{2}\right)^{T}\right)$ such that $\nu_{Z}\left(\left(-1, d z_{2}\right)^{T}\right)=0$. Then the process $\left(V_{t}^{x}\right)_{t \geq 0}$ defined by

$$
V_{t}^{x}=x+\int_{(0, t]} V_{s-}^{x} d U_{s}+L_{t}=x+\int_{(0, t]} g\left(V_{s-}^{x}\right) d Z_{s}, \quad t \geq 0,
$$

for $g(u)=(u, 1)$ is a Feller process whose generator $A^{V}$ has a domain containing

$$
S(\mathbb{R}):=\left\{f \in C_{0}^{2}(\mathbb{R}): \lim _{|x| \rightarrow \infty}\left(\left|x f^{\prime}(x)\right|+\left|x^{2} f^{\prime \prime}(x)\right|\right)=0\right\} .
$$

In particular $C_{c}^{\infty}(\mathbb{R}) \subset C_{c}^{2}(\mathbb{R}) \subset D\left(A^{V}\right)$. For any $f \in S(\mathbb{R})$ the generator can be written as

$$
\begin{aligned}
A^{V} f(x)= & f^{\prime}(x) g(x) \gamma_{Z}+\frac{1}{2} f^{\prime \prime}(x)\left(g(x) A_{Z} g(x)^{T}\right) \\
& +\int_{\mathbb{R}^{2}}\left(f(x+g(x) z)-f(x)-f^{\prime}(x) g(x) z \mathbb{1}_{|z| \leq 1}\right) \nu_{Z}(d z) \\
= & f^{\prime}(x)\left(x \gamma_{U}+\gamma_{L}\right)+\frac{1}{2} f^{\prime \prime}(x)\left(x^{2} \sigma_{U}^{2}+2 x \sigma_{U, L}+\sigma_{L}^{2}\right) \\
& +\int_{\mathbb{R}^{2}}\left(f\left(x+x z_{1}+z_{2}\right)-f(x)-f^{\prime}(x)\left(x z_{1}+z_{2}\right) \mathbb{1}_{|z| \leq 1}\right) \nu_{U, L}\left(d z_{1}, d z_{2}\right) .
\end{aligned}
$$

The spaces $S(\mathbb{R}), C_{c}^{2}(\mathbb{R})$ and $C_{c}^{\infty}(\mathbb{R})$ are cores for $A^{V}$.

Proof.

(i) Let us first establish the Feller property. It is well known that $V_{t}^{x}$ is a time homogenous Markov process (e.g. [4, Lem. 3.3]). By (2.4),$V_{t}^{x}$ is given by $V_{t}^{x}=\mathcal{E}(U)_{t}\left(x+\int_{(0, t]} \mathcal{E}(U)_{s-}^{-1} d \eta_{s}\right)$. Since $\mathcal{E}(U)_{t} \neq 0$ as a consequence of $\nu_{U}(\{-1\})=0$, we have $\lim _{|x| \rightarrow \infty}\left|V_{t}^{x}\right|=\infty$ and hence $\lim _{|x| \rightarrow \infty} f\left(V_{t}^{x}\right)=0$ for any $f \in C_{0}(\mathbb{R})$. By Lebesgue's dominated convergence theorem, this implies $T_{t} f(x)=E\left[f\left(V_{t}^{x}\right)\right] \rightarrow 0$ as $|x| \rightarrow \infty$. The fact that for bounded and continuous $f$ the mapping $x \mapsto E\left[f\left(V_{t}^{x}\right)\right]$ is continuous is obvious using dominated convergence. Thus $T_{t}$ maps $C_{0}(\mathbb{R})$ into $C_{0}(\mathbb{R})$ and $(\mathrm{F} 1)$ is shown. (F2) follows from (F1) and [26, Thm. $3.15]$, observing that for each $x \in \mathbb{R}, V^{x}$ satisfies $P\left(V_{0}^{x}=x\right)=1$ and $\left(V_{t}^{x}\right)_{t \geq 0}$ is adapted to the smallest filtration satisfying the usual hypotheses induced by $\left(\left(U_{t}, L_{t}\right)^{T}\right)_{t \geq 0}$, which is right continuous.

(ii) Before we prove (3.4), we give a bound for the integrand appearing in (3.4) which will be used throughout. Let $f \in S(\mathbb{R})$ and set

$$
K_{1}(f):=\sup _{y \in \mathbb{R}}\left\{\left|f^{\prime}(y)\right|(1+|y|)+\left|f^{\prime \prime}(y)\right|(1+|y|)^{2}\right\}<\infty \quad \text { and }
$$




$$
K_{2}:=\frac{1}{2} \sup _{y \in \mathbb{R}} \sup _{\zeta \in \mathbb{R}:|\zeta| \leq(1+|y|) / 2} \frac{(1+|y|)^{2}}{(1+|y+\zeta|)^{2}}<\infty .
$$

We claim that

$$
\begin{aligned}
& \left|f\left(x+x z_{1}+z_{2}\right)-f(x)-f^{\prime}(x)\left(x z_{1}+z_{2}\right) \mathbb{1}_{|z| \leq 1}\right| \\
& \quad \leq K_{1}(f) K_{2}|z|^{2} \mathbb{1}_{|z| \leq 1 / 2}+K_{1}(f)|z| \mathbb{1}_{1 / 2<|z| \leq 1}+2\|f\| \mathbb{1}_{|z|>1 / 2} \quad \forall z=\left(z_{1}, z_{2}\right)^{T} \in \mathbb{R}^{2}, x \in \mathbb{R} .
\end{aligned}
$$

Indeed, this is obvious for $|z|>1 / 2$ since $\left|x z_{1}+z_{2}\right| \leq \sqrt{1+x^{2}}|z| \leq(1+|x|)|z|$. For $|z| \leq 1 / 2$, by Taylor's theorem there is $\zeta \in \mathbb{R}$ with $0 \leq|\zeta| \leq\left|x z_{1}+z_{2}\right| \leq(1+|x|)|z| \leq$ $(1+|x|) / 2$ such that

$$
\begin{aligned}
& \left|f\left(x+x z_{1}+z_{2}\right)-f(x)-f^{\prime}(x)\left(x z_{1}+z_{2}\right)\right| \\
& \quad=2^{-1}\left|f^{\prime \prime}(x+\zeta)\right|\left(x z_{1}+z_{2}\right)^{2} \\
& \quad \leq 2^{-1}\left|f^{\prime \prime}(x+\zeta)(1+|x+\zeta|)^{2}\right| \frac{(1+|x|)^{2}}{(1+|x+\zeta|)^{2}}|z|^{2} \\
& \quad \leq K_{1}(f) K_{2}|z|^{2}
\end{aligned}
$$

which shows (3.6) also for $|z| \leq 1 / 2$. In particular, the right hand side of $(\underline{3.4})$ is in $C_{0}(\mathbb{R})$ for $f \in S(\mathbb{R})$ by Lebesgue's dominated convergence theorem.

(iii) Let us show (3.4). Let $f \in S(\mathbb{R})$, then by Itô's formula (e.g. [35, Thm. II.32]) we have

$$
\begin{aligned}
& f\left(V_{t}^{x}\right)-f\left(V_{0}^{x}\right) \\
& \quad=\int_{(0, t]} f^{\prime}\left(V_{s-}^{x}\right) d V_{s}^{x}+\frac{1}{2} \int_{(0, t]} f^{\prime \prime}\left(V_{s-}^{x}\right) d\left[V^{x}, V^{x}\right]_{s}^{c}+\sum_{0<s \leq t}\left(f\left(V_{s}^{x}\right)-f\left(V_{s-}^{x}\right)-f^{\prime}\left(V_{s}^{x}\right) \Delta V_{s}^{x}\right)
\end{aligned}
$$

and hence

$$
\begin{aligned}
T_{t} f(x)-f(x)= & E\left[f\left(V_{t}^{x}\right)-f\left(V_{0}^{x}\right)\right] \\
= & E\left[\int_{(0, t]} f^{\prime}\left(V_{s-}^{x}\right) d V_{s}^{x}+\sum_{0<s \leq t}\left(f\left(V_{s}^{x}\right)-f\left(V_{s-}^{x}\right)-f^{\prime}\left(V_{s-}^{x}\right) \Delta V_{s}^{x}\right)\right] \\
& +\frac{1}{2} E\left[\int_{(0, t]} f^{\prime \prime}\left(V_{s-}^{x}\right) d\left[V^{x}, V^{x}\right]_{s}^{c}\right] \\
=: & \mathrm{I}_{t}+\mathrm{II}_{t}, \quad \text { say. }
\end{aligned}
$$

Observe that $d V_{s}^{x}=g\left(V_{s-}^{x}\right) d Z_{s}$ and $\Delta V_{s}^{x}=g\left(V_{s-}^{x}\right) \Delta Z_{s}$. Since $Z$ is a Lévy process, by the Lévy-Itô decomposition (e.g. [2, Thm. 2.4.16]) we can write $Z_{t}=\gamma_{Z} t+M_{t}+$ $\sum_{0<s \leq t} \Delta Z_{s} \mathbb{1}_{\left|\Delta Z_{s}\right|>1}$, where $\left(M_{t}\right)_{t \geq 0}$ is a square integrable martingale with expectation 0 . Hence we obtain for the first term

$$
\begin{aligned}
\mathrm{I}_{t}= & E\left[\int_{(0, t]} f^{\prime}\left(V_{s-}^{x}\right) g\left(V_{s-}^{x}\right) \gamma_{Z} d s\right]+E\left[\int_{(0, t]} f^{\prime}\left(V_{s-}^{x}\right) g\left(V_{s-}^{x}\right) d M_{s}\right] \\
& +E\left[\sum_{0<s \leq t} f^{\prime}\left(V_{s-}^{x}\right) g\left(V_{s-}^{x}\right) \Delta Z_{s} \mathbb{1}_{\left|\Delta Z_{s}\right|>1}+\sum_{0<s \leq t}\left(f\left(V_{s}^{x}\right)-f\left(V_{s-}^{x}\right)-f^{\prime}\left(V_{s-}^{x}\right) g\left(V_{s-}^{x}\right) \Delta Z_{s}\right)\right] .
\end{aligned}
$$


Since $M$ is a square integrable martingale with expectation 0 and since $s \mapsto f^{\prime}\left(V_{s-}^{x}\right) g\left(V_{s-}^{x}\right)$ is bounded because of $f \in S(\mathbb{R})$, the process $t \mapsto \int_{(0, t]} f^{\prime}\left(V_{s-}^{x}\right) g\left(V_{s-}^{x}\right) d M_{s}$ is a martingale with expectation 0 (e.g. [32, Prop. 2.24]). Hence we conclude

$$
\begin{aligned}
\mathrm{I}_{t}= & \int_{(0, t]} E\left[f^{\prime}\left(V_{s-}^{x}\right) g\left(V_{s-}^{x}\right)\right] \gamma_{Z} d s \\
& +E\left[\sum_{0<s \leq t}\left(f\left(V_{s-}^{x}+g\left(V_{s-}^{x}\right) \Delta Z_{s}\right)-f\left(V_{s-}^{x}\right)-f^{\prime}\left(V_{s-}^{x}\right) g\left(V_{s-}^{x}\right) \Delta Z_{s} \mathbb{1}_{\left|\Delta Z_{s}\right| \leq 1}\right)\right] \\
= & \int_{(0, t]} E\left[f^{\prime}\left(V_{s-}^{x}\right) g\left(V_{s-}^{x}\right)\right] \gamma_{Z} d s \\
& +E\left[\int_{(0, t]} \int_{\mathbb{R}^{2}}\left(f\left(V_{s-}^{x}+g\left(V_{s-}^{x}\right) z\right)-f\left(V_{s-}^{x}\right)-f^{\prime}\left(V_{s-}^{x}\right) g\left(V_{s-}^{x}\right) z \mathbb{1}_{|z| \leq 1}\right) \nu_{Z}(d z) d s\right],
\end{aligned}
$$

where we used the compensation formula (e.g. [24, Thm. 4.4]), which may be applied since $E \int_{(0, t]} \int_{\mathbb{R}^{2}}\left|f\left(V_{s-}^{x}+g\left(V_{s-}^{x}\right) z\right)-f\left(V_{s-}^{x}\right)-f^{\prime}\left(V_{s-}^{x}\right) g\left(V_{s-}^{x}\right) z \mathbb{1}_{|z| \leq 1}\right| \nu_{Z}(d z) d s$ is finite by (3.6) $)$. Using the continuity of $s \mapsto V_{s}^{x}$ at $s=0$ and again the bound from (3.6), it follows from Lebesgue's dominated convergence theorem that

$$
\lim _{t \rightarrow 0} t^{-1} \mathrm{I}_{t}=f^{\prime}(x) g(x) \gamma_{Z}+\int_{\mathbb{R}^{2}}\left(f(x+g(x) z)-f(x)-f^{\prime}(x) g(x) z \mathbb{1}_{|z| \leq 1}\right) \nu_{Z}(d z) .
$$

For the second term in Equation (3.7) observe that by [18, Eq. (4)]

$$
\begin{aligned}
{\left[V^{x}, V^{x}\right]_{s}^{c} } & =\left[x+\int_{(0, \cdot]} g\left(V_{u-}^{x}\right) d Z_{u}, x+\int_{(0, \cdot]} g\left(V_{u-}^{x}\right) d Z_{u}\right]_{s}^{c} \\
& =\int_{(0, s]} g\left(V_{u-}^{x}\right) d\left[Z, Z^{T}\right]_{u}^{c} g\left(V_{u-}^{x}\right)^{T}
\end{aligned}
$$

and since $\left[Z, Z^{T}\right]_{u}^{c}=A_{Z} u$ it follows

$$
\mathrm{II}_{t}=\frac{1}{2} E\left[\int_{(0, t]} f^{\prime \prime}\left(V_{s-}^{x}\right) g\left(V_{u-}^{x}\right) A_{Z} g\left(V_{u-}^{x}\right)^{T} d u\right] .
$$

Together with the obtained formula for $\mathrm{I}_{t}$, and inserting the definition of $g$ and $Z$, this shows that $\lim _{t \rightarrow 0} t^{-1}\left(\mathrm{I}_{t}+\mathrm{II}_{t}\right)$ is given by the right hand side of (3.4). Since $V$ is a Feller process, and since the right hand side of $(3.4)$ is in $C_{0}(\mathbb{R})$ for $f \in S(\mathbb{R})$ by $(3.6)$, this pointwise limit is actually uniform in $x$ (e.g. [36, Lem. 31.7]), so that $S(\mathbb{R})$ is contained in the domain of the generator of $V$ and that $A^{V} f$ is given by (3.4) for all $f \in S(\mathbb{R})$.

(iv) We now show that $S(\mathbb{R})$ is a core for $A^{V}$ under the extra assumption that $E U_{1}^{2}<$ $\infty$ and $E L_{1}^{2}<\infty$. Denote $A_{t}=\mathcal{E}(U)_{t}$ and $B_{t}=\mathcal{E}(U)_{t} \int_{(0, t]} \mathcal{E}(U)_{s}^{-1} d \eta_{s}$. Then $B_{t} \stackrel{d}{=}$ $\int_{(0, t]} \mathcal{E}(U)_{s-} d L_{s}$ by [4, Lem. 3.1]. Then $E A_{t}^{2}<\infty$ and $E B_{t}^{2}<\infty$ as a consequence of 
Proposition 3.1 and Lemma 6.1 in [3] together with [36, Thm. 25.18]. We conclude that $\frac{\partial^{2}}{\partial x^{2}} T_{t} f(x)$ exists for $f \in S(\mathbb{R})$ and that

$$
\begin{aligned}
\frac{\partial}{\partial x} T_{t} f(x) & =\frac{\partial}{\partial x} E\left[f\left(A_{t} x+B_{t}\right)\right]=E\left[A_{t} f^{\prime}\left(A_{t} x+B_{t}\right)\right] \quad \text { and } \\
\frac{\partial^{2}}{\partial x^{2}} T_{t} f(x) & =\frac{\partial^{2}}{\partial x^{2}} E\left[f\left(A_{t} x+B_{t}\right)\right]=E\left[A_{t}^{2} f^{\prime \prime}\left(A_{t} x+B_{t}\right)\right] .
\end{aligned}
$$

Since $E A_{t}^{2}<\infty$, the mapping $x \mapsto \frac{\partial^{2}}{\partial x^{2}} T_{t} f(x)$ is obviously continuous, so that $T_{t} S(\mathbb{R}) \subset$ $C^{2}(\mathbb{R}) \cap C_{0}(\mathbb{R})$. Using that $E\left|B_{t}\right|<\infty$ and $\lim _{|y| \rightarrow \infty}\left|y f^{\prime}(y)\right|=0$ for $f \in S(\mathbb{R})$, we further see by dominated convergence that

$$
\begin{aligned}
\left|x \frac{\partial}{\partial x} T_{t} f(x)\right| & \leq E\left[\left|A_{t} x f^{\prime}\left(A_{t} x+B_{t}\right)\right|\right] \\
& \leq E\left[\left|\left(A_{t} x+B_{t}\right) f^{\prime}\left(A_{t} x+B_{t}\right)\right|\right]+E\left[\left|B_{t} f^{\prime}\left(A_{t} x+B_{t}\right)\right|\right] \\
& \rightarrow 0, \quad \text { as }|x| \rightarrow \infty
\end{aligned}
$$

In the same way one can check that $\left|x^{2} \frac{\partial^{2}}{\partial x^{2}} T_{t} f(x)\right| \rightarrow 0$ as $|x| \rightarrow \infty$ such that $T_{t} S(\mathbb{R}) \subset$ $S(\mathbb{R})$. By [13, Prop. 1.3.3] we thus obtain that $S(\mathbb{R})$ is a core for $A^{V}$, provided that $E U_{t}^{2}<\infty$ and $E L_{t}^{2}<\infty$.

(v) Now we drop the assumption that $E U_{1}^{2}+E L_{1}^{2}<\infty$ and show that $S(\mathbb{R})$ is a core for $A^{V}$. Similarly to the proof of Theorem 3.1 in Sato and Yamazato [38], for $f \in S(\mathbb{R})$ denote the right hand side of (3.4) by $G f(x)$ and define

$$
\begin{aligned}
G_{0} f(x):= & f^{\prime}(x)\left(x \gamma_{U}+\gamma_{L}\right)+\frac{1}{2} f^{\prime \prime}(x)\left(x^{2} \sigma_{U}^{2}+2 x \sigma_{U, L}+\sigma_{L}^{2}\right) \\
& +\int_{\left\{z \in \mathbb{R}^{2}:|z| \leq 1\right\}}\left(f\left(x+x z_{1}+z_{2}\right)-f(x)-f^{\prime}(x)\left(x z_{1}+z_{2}\right) \mathbb{1}_{|z| \leq 1}\right) \nu_{U, L}\left(d z_{1}, d z_{2}\right) .
\end{aligned}
$$

For $f \in C_{0}(\mathbb{R})$, denote further

$$
W f(x):=\int_{\left\{z \in \mathbb{R}^{2}:|z|>1\right\}}\left(f\left(x+x z_{1}+z_{2}\right)-f(x)\right) \nu_{U, L}\left(d z_{1}, d z_{2}\right) .
$$

Then $W: C_{0}(\mathbb{R}) \rightarrow C_{0}(\mathbb{R})$ is a bounded linear operator, and from (iii) we know that $A^{V} f=G f=G_{0} f+W f$ for $f \in S(\mathbb{R})$. Consider the process $V_{(0)}$ defined by $V_{(0), t}^{x}=$ $x+\int_{(0, t]} V_{(0), s-}^{x} d \tilde{U}_{s}+\tilde{L}_{t}$, where $(\tilde{U}, \tilde{L})^{T}$ is a Lévy process with characteristic triplet $\left(\gamma_{Z}, A_{Z}, \mathbb{1}_{|z| \leq 1} \nu_{Z}(d z)\right)$. Again by (iii), $G_{0} f=A^{V_{(0)} f}$ for $f \in S(\mathbb{R})$, and from (iv) we know that $S(\mathbb{R})$ is a core for $A^{V_{(0)}}$, so that the closure $\overline{G_{0}}$ of $G_{0}$ is $A^{V_{(0)}}$, in particular $D\left(\overline{G_{0}}\right)=D\left(A^{V_{(0)}}\right)$. Since $G f=G_{0} f+W f$ for $f \in S(\mathbb{R})$ and $W$ is bounded, it follows that the closure $\bar{G}$ of $G$ satisfies $\bar{G}=\overline{G_{0}}+W$, in particular $D\left(A^{V_{(0)}}\right)=D\left(\overline{G_{0}}\right)=D(\bar{G})$. Since $A^{V}$ is a closed operator, we further know that $D(\bar{G}) \subset D\left(A^{V}\right)$ and that $A^{V}$ is a closed extension of $\bar{G}$. From the Hille-Yosida theorem (e.g. [13, Thm. 1.2.6]) it follows that 
for every $\lambda>0, \lambda \mathrm{Id}-\overline{G_{0}}: D\left(A^{V_{(0)}}\right)=D\left(\overline{G_{0}}\right) \rightarrow C_{0}(\mathbb{R}), f \mapsto \lambda f-\overline{G_{0}} f$ is a bijection with bounded inverse (the resolvent) satisfying $\left\|\left(\lambda \mathrm{Id}-\overline{G_{0}}\right)^{-1}\right\| \leq \lambda^{-1}$. For $\lambda_{0}>\|W\|$, it then follows from a perturbation result for closed linear operators (e.g. [19, Thm. IV.1.16]), that also $\lambda_{0} \mathrm{Id}-\bar{G}=\lambda_{0} \mathrm{Id}-\overline{G_{0}}-W: D(\bar{G})=D\left(\overline{G_{0}}\right) \rightarrow C_{0}(\mathbb{R})$ is a bijection with bounded inverse. Since $A^{V}$ is a closed extension of $\bar{G}$ and also $\lambda_{0} \operatorname{Id}-A^{V}: D\left(A^{V}\right) \rightarrow C_{0}(\mathbb{R})$ is a bijection (e.g. [13, Prop. 1.2.1]), we must have $D(\bar{G})=D\left(A^{V}\right)$ and hence $\bar{G}=A^{V}$. This shows that $S(\mathbb{R})$ is a core for $A^{V}$.

(vi) Finally, we show that $C_{c}^{2}(\mathbb{R})$ and $C_{c}^{\infty}(\mathbb{R})$ are cores for $A^{V}$. Let $h$ be a function in $C_{c}^{\infty}$ with $h(x)=1$ if $|x| \leq 1$ and $h(x)=0$ if $|x| \geq 2$. Define $h_{n}(x)=h(x / n)$ and for any $f \in S(\mathbb{R})$ set $f_{n}(x)=f(x) h_{n}(x)$. Then $f_{n} \in C_{c}^{2}(\mathbb{R})$ and we obtain that $f_{n} \rightarrow f, f_{n}^{\prime} \rightarrow f^{\prime}$, $f_{n}^{\prime \prime} \rightarrow f^{\prime \prime}, x f_{n}^{\prime}(x) \rightarrow x f^{\prime}(x), x f_{n}^{\prime \prime}(x) \rightarrow x f^{\prime \prime}(x)$ and $x^{2} f_{n}^{\prime \prime}(x) \rightarrow x^{2} f^{\prime \prime}(x)$ uniformly in $x$ as $n \rightarrow \infty$. In particular, with $K_{1}(\cdot)$ as defined in (3.5), we see that $K_{1}\left(f_{n}\right)$ is bounded in $n$ and hence we conclude with (3.6) that $A^{V} f_{n} \rightarrow A^{V} f$ uniformly as $n \rightarrow \infty$. This shows that $C_{c}^{2}(\mathbb{R})$ is a core for $A^{V}$. Finally, for $f \in C_{c}^{2}(\mathbb{R})$ there are functions $g_{n} \in C_{c}^{\infty}(\mathbb{R})$ with uniformly bounded supports such that $g_{n} \rightarrow f, g_{n}^{\prime} \rightarrow f^{\prime}$ and $g_{n}^{\prime \prime} \rightarrow f^{\prime \prime}$ uniformly as $n \rightarrow \infty$, hence also $x g_{n}^{\prime}(x) \rightarrow x f^{\prime}(x), x g_{n}^{\prime \prime}(x) \rightarrow x f^{\prime \prime}(x)$ and $x^{2} g_{n}^{\prime \prime}(x) \rightarrow x^{2} f^{\prime \prime}(x)$ uniformly in $x$ as $n \rightarrow \infty$. Again, this gives $A^{V} g_{n} \rightarrow A^{V} f$ uniformly as $n \rightarrow \infty$ so that $C_{c}^{\infty}$ is a core for $A^{V}$.

The following corollary is immediate from Theorem 3.1 .

Corollary 3.2. In the setting of Theorem 3.1, if $U$ and $L$ are additionally independent, Equation (3.4) simplifies to

$$
\begin{aligned}
A^{V} f(x)= & f^{\prime}(x)\left(x \gamma_{U}+\gamma_{L}\right)+\frac{1}{2} f^{\prime \prime}(x)\left(x^{2} \sigma_{U}^{2}+\sigma_{L}^{2}\right) \\
& +\int_{\mathbb{R}}\left(f(x+x y)-f(x)-f^{\prime}(x) x y \mathbb{1}_{|y| \leq 1}\right) \nu_{U}(d y) \\
& +\int_{\mathbb{R}}\left(f(x+y)-f(x)-f^{\prime}(x) y \mathbb{1}_{|y| \leq 1}\right) \nu_{L}(d y) \\
= & A^{L} f(x)+f^{\prime}(x) x \gamma_{U}+\frac{1}{2} f^{\prime \prime}(x) x^{2} \sigma_{U}^{2} \\
& +\int_{\mathbb{R}}\left(f(x+x y)-f(x)-f^{\prime}(x) x y \mathbb{1}_{|y| \leq 1}\right) \nu_{U}(d y) .
\end{aligned}
$$

Corollary 3.3. Let $\left(\xi_{t}\right)_{t \geq 0}$ and $\left(\eta_{t}\right)_{t \geq 0}$ be two independent Lévy processes and let $\left(V_{t}^{x}\right)_{t \geq 0}$ be the generalized Ornstein-Uhlenbeck process driven by $(\xi, \eta)^{T}$ with starting point $x$ as defined in (2.1). Then $\left(V_{t}^{x}\right)_{t \geq 0}$ is a Feller process whose generator has a domain containing $S(\mathbb{R})$, and $S(\mathbb{R}), C_{c}^{2}(\mathbb{R})$ and $C_{c}^{\infty}(\mathbb{R})$ are cores for $A^{V}$. For any $f \in S(\mathbb{R})$ the generator can be written as

$$
\begin{aligned}
A^{V} f(x)= & A^{\eta} f(x)-f^{\prime}(x) x \gamma_{\xi}+\frac{1}{2}\left(f^{\prime \prime}(x) x^{2}+f^{\prime}(x) x\right) \sigma_{\xi}^{2} \\
& +\int_{\mathbb{R}}\left(f\left(x e^{-y}\right)-f(x)+f^{\prime}(x) x y \mathbb{1}_{|y| \leq 1}\right) \nu_{\xi}(d y) .
\end{aligned}
$$


If $f \in S(\mathbb{R})$ and $f(0)=0$, define $\tilde{f}(x)=f\left(e^{x}\right)$ and $\tilde{\tilde{f}}(x)=f\left(-e^{x}\right)$. Then $\tilde{f}, \tilde{\tilde{f}} \in C_{0}^{2}(\mathbb{R}) \subset$ $D\left(A^{-\xi}\right)$, and for such $f$ Equation (3.9) can be rewritten as

$$
A^{V} f(x)=A^{\eta} f(x)+A^{-\xi} \tilde{f}(\log x) \mathbb{1}_{x>0}+A^{-\xi} \tilde{\tilde{f}}(\log |x|) \mathbb{1}_{x<0} .
$$

Proof. Since $\left(V_{t}^{x}\right)_{t \geq 0}$ fulfills (3.3) for $(U, L)^{T}$ defined in (2.3) the Feller property as well as the statements on the domain and cores of the generator follow directly from Theorem 3.1 , Also observe from (2.3) that in the independent case we have $\eta_{t}=L_{t}$ and thus $A^{\eta}=A^{L}$ whereas the relation between $\xi$ and $U$ yields $\nu_{U}((-\infty,-1])=0$. In [4, Lem. 3.4] we have computed the characteristic triplet of $\xi$ in terms of the characteristic triplet of $U$ (the $\hat{U}$ used there is equal to $\xi$ whenever $\left.\nu_{U}((-\infty,-1])=0\right)$. Using these relations one obtains (3.9) from (3.8) by standard computations.

Finally the fact that $\tilde{f}, \tilde{\tilde{f}} \in C_{0}^{2}(\mathbb{R})$ if $f \in S(\mathbb{R})$ such that $f(0)=0$ and the validity of (3.10) may be checked directly from (3.1) using the definitions of $\tilde{f}$ and $\tilde{\tilde{f}}$.

Remark 3.4. In [23] the exponential functional for independent processes $\xi$ and $\eta$ is studied. Under the condition of finite first moments of $\xi$ and $\eta$, the authors prove that for suitable functions $f$ with support on the positive half line the generator of the GOU process can be written as

$$
A^{V} f(x)=A^{-\xi} \tilde{f}(\log x)+A^{\eta} f(x)
$$

where $\tilde{f}(x)=f\left(e^{x}\right)$ and $A^{-\xi}$ and $A^{\eta}$ are the generators of $-\xi$ and $\eta$ respectively. Remark that the $\xi$ used by the authors corresponds to $-\xi$ in our notation. The formula (3.11) for positive $x$ is also obtained in [9, Proof of Thm. 1].

\section{Relations between the exponential functional and the driving Lévy processes}

It is basic knowledge in the theory of Markov processes (see e.g. [13, Prop. 4.9.2]), that if $\mu$ is an invariant measure for the Markov process $X$ with strongly continuous contraction semigroup $T_{t}$ and generator $A$, i.e. if $\mu(B)=\int \mu_{t}(x, B) \mu(d x)$ for all Borel sets $B$, then

$$
\int_{\mathbb{R}^{d}} A f(y) \mu(d y)=0 \quad \forall f \in D(A)
$$

Conversely, if (4.1) holds, $\mu$ is an invariant measure under some additional conditions. In the special case of Feller processes Equation (4.1) holds if and only if $\mu$ is an invariant measure of the corresponding process $X$ [26, Thm. 3.37].

In [10] and [11] the authors make use of Equation (4.1) to obtain the density of a specific stationary generalized Ornstein-Uhlenbeck process. More precisely they obtain the density of the exponential functional in the special case that $\xi$ is a Brownian motion with drift and $\eta$ is deterministic. 
Let $\left(V_{t}\right)_{t \geq 0}$ be a GOU process as defined in (2.1) or even a process as defined in (2.4), fulfilling the SDE (2.2), with $\nu_{U}(\{-1\})=0$. Assume that $U$ and $L$ are independent, i.e. the generator of $\left(V_{t}\right)_{t \geq 0}$ is given by (3.8) for $f \in S(\mathbb{R})$. Let $\mu=\mathcal{L}\left(V_{\infty}\right)$ be the invariant measure of $\left(V_{t}\right)_{t \geq 0}$, assuming its existence. Then by (4.1) we obtain for any $f \in S(\mathbb{R}) \subset D\left(A^{V}\right)$

$$
\begin{aligned}
0= & \int_{\mathbb{R}} A^{V} f(x) \mu(d x) \\
= & \int_{\mathbb{R}} A^{L} f(x) \mu(d x)+\gamma_{U} \int_{\mathbb{R}} f^{\prime}(x) x \mu(d x)+\frac{\sigma_{U}^{2}}{2} \int_{\mathbb{R}} f^{\prime \prime}(x) x^{2} \mu(d x) \\
& +\int_{\mathbb{R}} \int_{\mathbb{R} \backslash\{-1\}}\left(f(x+x y)-f(x)-f^{\prime}(x) x y \mathbb{1}_{|y| \leq 1}\right) \nu_{U}(d y) \mu(d x) .
\end{aligned}
$$

This and the previous results allow to establish relationships between the characteristic functions of $V_{\infty}, U$ and $L$, as done in the following. Recall that $\psi_{X}(u)=\log E\left[e^{i u X_{1}}\right]$ is the characteristic exponent of the Lévy process $X$.

Theorem 4.1. Let $\left(U_{t}\right)_{t \geq 0}$ and $\left(L_{t}\right)_{t \geq 0}$ be two independent Lévy processes with $\nu_{U}(\{-1\})=$ 0 and such that $V_{\infty}=\int_{0}^{\infty} \mathcal{E}(U)_{s-} d L_{s}$ converges to a finite random variable. Then $\mu=$ $\mathcal{L}\left(V_{\infty}\right)$ is the invariant law of the process $\left(V_{t}\right)_{t \geq 0}$ defined by (2.4).

Let $h \in C_{c}^{\infty}(\mathbb{R})$ be such that $h(x)=1$ for $|x| \leq 1$ and $h(x)=0$ for $|x| \geq 2$ and set $h_{n}(x):=h\left(\frac{x}{n}\right)$ and $f(x)=e^{i u x}, f_{n}(x)=f(x) h_{n}(x)$ for $u \in \mathbb{R}$. Then

$$
\begin{aligned}
\psi_{L}(u) \phi_{V_{\infty}}(u)= & \lim _{n \rightarrow \infty}\left(-\gamma_{U} \int_{\mathbb{R}} x f_{n}^{\prime}(x) \mu(d x)-\frac{\sigma_{U}^{2}}{2} \int_{\mathbb{R}} x^{2} f_{n}^{\prime \prime}(x) \mu(d x)\right. \\
& \left.-\int_{\mathbb{R}} \int_{\mathbb{R}}\left(f_{n}(x+x y)-f_{n}(x)-x y f_{n}^{\prime}(x) \mathbb{1}_{|y| \leq 1}\right) \nu_{U}(d y) \mu(d x)\right) .
\end{aligned}
$$

If additionally $E\left[V_{\infty}^{2}\right]=\int_{\mathbb{R}} x^{2} \mu(d x)<\infty$, then

$$
\begin{aligned}
\psi_{L}(u) \phi_{V_{\infty}}(u)= & -i u \gamma_{U} E\left[V_{\infty} e^{i u V_{\infty}}\right]+\frac{\sigma_{U}^{2} u^{2}}{2} E\left[V_{\infty}^{2} e^{i u V_{\infty}}\right] \\
& -\int_{\mathbb{R}}\left(\phi_{V_{\infty}}(u(1+y))-\phi_{V_{\infty}}(u)-i u E\left[V_{\infty} e^{i u V_{\infty}}\right] y \mathbb{1}_{|y| \leq 1}\right) \nu_{U}(d y) \\
= & -u \gamma_{U} \phi_{V_{\infty}}^{\prime}(u)-\frac{\sigma_{U}^{2} u^{2}}{2} \phi_{V_{\infty}}^{\prime \prime}(u) \\
& -\int_{\mathbb{R}}\left(\phi_{V_{\infty}}(u(1+y))-\phi_{V_{\infty}}(u)-u \phi_{V_{\infty}}^{\prime}(u) y \mathbb{1}_{|y| \leq 1}\right) \nu_{U}(d y) \\
= & -E\left[e^{i u V_{\infty}} \psi_{U}\left(u V_{\infty}\right)\right]
\end{aligned}
$$

Equation (4.6) can also be written in the compact form

$$
E\left[\left(\psi_{U}\left(u V_{\infty}\right)+\psi_{L}(u)\right) e^{i u V_{\infty}}\right]=0 \quad \forall u \in \mathbb{R} .
$$

For the proof we need the following lemma. We use the notation $S(\mathbb{R} ; \mathbb{C})$ to denote the class of complex valued functions $f: \mathbb{R} \rightarrow \mathbb{C}$ such that $\Re(f) \in S(\mathbb{R})$ and $\Im(f) \in S(\mathbb{R})$. Spaces like $C_{c}^{\infty}(\mathbb{R} ; \mathbb{C})$ are defined similarly. For a generator $A$ we also write

$$
D(A ; \mathbb{C}):=\left\{f \in C_{0}(\mathbb{R} ; \mathbb{C}): \Re(f), \Im(f) \in D(A)\right\} .
$$


It is clear that (3.8) and hence (4.2) remain valid for complex valued functions $f \in S(\mathbb{R} ; \mathbb{C})$.

Lemma 4.2. Let $\left(L_{t}\right)_{t \geq 0}$ be a Lévy process in $\mathbb{R}$ with generator $A^{L}$, $\mu$ a fixed finite measure on $\left(\mathbb{R}, \mathcal{B}_{1}\right)$ and define $h, h_{n}, f$ and $f_{n}$ as in Theorem 4.1. Then $f_{n} \in C_{c}^{\infty}(\mathbb{R} ; \mathbb{C}) \subset D\left(A^{L} ; \mathbb{C}\right)$ and

$$
\lim _{n \rightarrow \infty} \int_{\mathbb{R}} A^{L} f_{n}(x) \mu(d x)=\psi_{L}(u) \int_{\mathbb{R}} e^{i u x} \mu(d x)=\psi_{L}(u) \widehat{\mu}(u) .
$$

Proof. It is clear that $f_{n} \in C_{c}^{\infty}(\mathbb{R} ; \mathbb{C})$. From (3.1) we obtain

$$
\begin{aligned}
\int_{\mathbb{R}} A^{L} f_{n}(x) \mu(d x)= & \gamma_{L} \int_{\mathbb{R}} f_{n}^{\prime}(x) \mu(d x)+\frac{\sigma_{L}^{2}}{2} \int_{\mathbb{R}} f_{n}^{\prime \prime}(x) \mu(d x) \\
& +\int_{\mathbb{R}} \int_{\mathbb{R}}\left(f_{n}(x+y)-f_{n}(x)-f_{n}^{\prime}(x) y \mathbb{1}_{|y| \leq 1}\right) \nu_{L}(d y) \mu(d x) .
\end{aligned}
$$

By Taylor's formula, there are $\zeta_{1}, \zeta_{2} \in[-|y|,|y|]$ such that

$$
\begin{aligned}
& \left|f_{n}(x+y)-f_{n}(x)-f_{n}^{\prime}(x) y \mathbb{1}_{|y| \leq 1}\right| \\
& \quad \leq\left|f_{n}(x+y)-f_{n}(x)\right| \mathbb{1}_{|y|>1}+2^{-1}\left[\left|\left(\Re f_{n}^{\prime \prime}\right)\left(x+\zeta_{1}\right)\right|+\left|\left(\Im f_{n}^{\prime \prime}\right)\left(x+\zeta_{2}\right)\right|\right] y^{2} \mathbb{1}_{|y| \leq 1} \\
& \quad \leq 2\left\|f_{n}\right\| \mathbb{1}_{|y|>1}+\left\|f_{n}^{\prime \prime}\right\| y^{2} \mathbb{1}_{|y| \leq 1} .
\end{aligned}
$$

Computing the first two derivatives of $f_{n}$ one easily sees that they are uniformly bounded in $n$. Since additionally $\lim _{n \rightarrow \infty} f_{n}^{\prime}(x)=i u e^{i u x}$ and $\lim _{n \rightarrow \infty} f_{n}^{\prime \prime}(x)=-u^{2} e^{i u x}$ we obtain via dominated convergence

$$
\begin{aligned}
\lim _{n \rightarrow \infty} \int_{\mathbb{R}} A^{L} f_{n}(x) \mu(d x)= & \gamma_{L} \int_{\mathbb{R}} i u e^{i u x} \mu(d x)-\frac{\sigma_{L}^{2}}{2} \int_{\mathbb{R}} u^{2} e^{i u x} \mu(d x) \\
& +\int_{\mathbb{R}} \int_{\mathbb{R}}\left(e^{i u(x+y)}-e^{i u x}-i u e^{i u x} y \mathbb{1}_{|y| \leq 1}\right) \nu_{L}(d y) \mu(d x),
\end{aligned}
$$

which gives the claim.

Proof of Theorem 4.1. Since $\int_{0}^{t} \mathcal{E}(U)_{s-} d L_{s}$ converges almost surely to the finite random variable $V_{\infty}$ as $t \rightarrow \infty, \mu=\mathcal{L}\left(V_{\infty}\right)$ is the unique stationary marginal distribution and hence invariant law of $V$ by [4, Thms. 2.1 and 3.6]. Equation (4.3) then follows directly from (4.1), (4.2) and Lemma 4.2.

To show (4.4), observe that by Taylor's formula there are $\zeta_{1}, \zeta_{2} \in[-|x y|,|x y|]$ such that

$$
\begin{aligned}
& \left|f_{n}(x+x y)-f_{n}(x)-x y f_{n}^{\prime}(x) \mathbb{1}_{|y| \leq 1}\right| \\
& \quad \leq\left|f_{n}(x+x y)-f_{n}(x)\right| \mathbb{1}_{|y|>1}+2^{-1}\left[\left|\left(\Re f_{n}^{\prime \prime}\right)\left(x+\zeta_{1}\right)\right|+\left|\left(\Im f_{n}^{\prime \prime}\right)\left(x+\zeta_{2}\right)\right|\right] x^{2} y^{2} \mathbb{1}_{|y| \leq 1} \\
& \quad \leq 2\left\|f_{n}\right\| \mathbb{1}_{|y|>1}+\left\|f_{n}^{\prime \prime}\right\| x^{2} y^{2} \mathbb{1}_{|y| \leq 1} .
\end{aligned}
$$

Equation (4.4) then follows directly from (4.3) by dominated convergence and Fubini's theorem, observing as in the proof of Lemma 4.2 that $f_{n}$ and its first two derivatives are uniformly bounded in $n$. Finally, Equations (4.5) and (4.6) are immediate consequences of (4.4). 
For GOU processes driven by $(\xi, \eta)$, Theorem 4.1 gives the following.

Corollary 4.3. Let $\left(\xi_{t}\right)_{t \geq 0}$ and $\left(\eta_{t}\right)_{t \geq 0}$ be two independent Lévy processes such that $V_{\infty}=$ $\int_{0}^{\infty} e^{-\xi_{s-}} d \eta_{s}$ converges to a finite random variable. Then $\mu=\mathcal{L}\left(V_{\infty}\right)$ is the invariant law of the GOU process $\left(V_{t}\right)_{t \geq 0}$ driven by $(\xi, \eta)^{T}$ as defined in (2.1).

Let $h \in C_{c}^{\infty}(\mathbb{R})$ such that $h(x)=1$ for $|x| \leq 1$ and $h(x)=0$ for $|x| \geq 2$ and set $h_{n}(x):=h\left(\frac{x}{n}\right)$ and $f(x)=e^{i u x}, f_{n}(x)=f(x) h_{n}(x)$ for $u \in \mathbb{R}$. Then

$$
\begin{aligned}
\psi_{\eta}(u) \phi_{V_{\infty}}(u)= & \lim _{n \rightarrow \infty}\left(\gamma_{\xi} \int_{\mathbb{R}} x f_{n}^{\prime}(x) \mu(d x)-\frac{\sigma_{\xi}^{2}}{2} \int_{\mathbb{R}}\left(x^{2} f_{n}^{\prime \prime}(x)+x f_{n}^{\prime}(x)\right) \mu(d x)\right. \\
& \left.-\int_{\mathbb{R}} \int_{\mathbb{R}}\left(f_{n}\left(x e^{-y}\right)-f_{n}(x)+x y f_{n}^{\prime}(x) \mathbb{1}_{|y| \leq 1}\right) \nu_{\xi}(d y) \mu(d x)\right) .
\end{aligned}
$$

If additionally $E\left[V_{\infty}^{2}\right]<\infty$, then

$$
\begin{aligned}
\psi_{\eta}(u) \phi_{V_{\infty}}(u)= & \gamma_{\xi} u \phi_{V_{\infty}}^{\prime}(u)-\frac{\sigma_{\xi}^{2}}{2}\left(u^{2} \phi_{V_{\infty}}^{\prime \prime}(u)+u \phi_{V_{\infty}}^{\prime}(u)\right) \\
& -\int_{\mathbb{R}}\left(\phi_{V_{\infty}}\left(u e^{-y}\right)-\phi_{V_{\infty}}(u)+u y \phi_{V_{\infty}}^{\prime}(u) \mathbb{1}_{|y| \leq 1}\right) \nu_{\xi}(d y) .
\end{aligned}
$$

Proof. This follows directly from Theorem 4.1 and the relations between $(U, L)$ and $(\xi, \eta)$ as given in (2.3) and [4, Lem. 3.4], or alternatively using (3.9) and arguments as in the proof of Theorem 4.1.

Observe that for $\xi$ being a compound Poisson process Equation (4.8) immediately gives (1.2).

Remark 4.4. Carmona [9, Thm. 2] obtains a formula related to (4.8) under certain, more restrictive assumptions. In particular, it is assumed in [9] that $e^{\xi_{t}}$ admits a strictly positive density on some interval $\left(0, r_{t}\right)$ for some $r_{t}>0$. In the special case that $\eta$ is a compound Poisson process without negative jumps and $\xi$ is a Brownian motion with drift, formula (4.8) has already been obtained by Nilsen and Paulsen [33, Prop. 2], stated for Laplace transforms.

Remark 4.5. Let $\eta$ be a subordinator, $\xi$ a Lévy process independent of $\eta$, and suppose that $V_{\infty}:=\int_{0}^{\infty} e^{-\xi_{s-}} d \eta_{s}$ is almost surely finite. Then $V_{\infty} \geq 0$, and we can also use Laplace transforms in the above derivation. More precisely, let $(U, L)^{T}$ be given by (2.3), so that $L=\eta$ by independence and $e^{-\xi_{t}}=\mathcal{E}(U)_{t}$, where $\nu_{U}((-\infty,-1])=0$. Denote the Laplace transforms of $\eta=L$ and $V_{\infty}$ for $u \geq 0$ by $\mathbb{L}_{\eta}(u)=\mathbb{L}_{L}(u)=E\left[e^{-u \eta_{1}}\right]=\phi_{\eta}(i u)$ and $\mathbb{L}_{V_{\infty}}(u)=E\left[e^{-u V_{\infty}}\right]$, respectively. Let $f$ be a function in $S(\mathbb{R})$ with $f(x)=e^{-u x}, x \geq 0$, then $f$ is in $D\left(A^{V}\right)$ for $u>0$ and a direct computation starting from (4.2) yields the following analogues of (4.4) and (4.8) without any further moment restrictions on the distribution of $V_{\infty}$.

$$
\begin{aligned}
& \log \mathbb{L}_{L}(u)=\log \mathbb{L}_{\eta}(u) \\
& \quad=u \gamma_{U} \frac{E\left[V_{\infty} e^{-u V_{\infty}}\right]}{\mathbb{L}_{V_{\infty}}(u)}-\frac{\sigma_{U}^{2} u^{2}}{2} \frac{E\left[V_{\infty}^{2} e^{-u V_{\infty}}\right]}{\mathbb{L}_{V_{\infty}}(u)}
\end{aligned}
$$




$$
\begin{aligned}
& -\int_{(-1, \infty)}\left(\frac{\mathbb{L}_{V_{\infty}}(u(1+y))}{\mathbb{L}_{V_{\infty}}(u)}-1+u \frac{E\left[V_{\infty} e^{-u V_{\infty}}\right]}{\mathbb{L}_{V_{\infty}}(u)} y \mathbb{1}_{|y| \leq 1}\right) \nu_{U}(d y) \\
= & -u \gamma_{\xi} \frac{E\left[V_{\infty} e^{-u V_{\infty}}\right]}{\mathbb{L}_{V_{\infty}}(u)}-\frac{\sigma_{\xi}^{2}}{2}\left(\frac{E\left[V_{\infty}^{2} e^{-u V_{\infty}}\right]}{\mathbb{L}_{V_{\infty}}(u)} u^{2}-\frac{E\left[V_{\infty} e^{-u V_{\infty}}\right]}{\mathbb{L}_{V_{\infty}}(u)} u\right) \\
& -\int_{\mathbb{R}}\left(\frac{\mathbb{L}_{V_{\infty}}\left(u e^{-y}\right)}{\mathbb{L}_{V_{\infty}}(u)}-1-u \frac{E\left[V_{\infty} e^{-u V_{\infty}}\right]}{\mathbb{L}_{V_{\infty}}(u)} y \mathbb{1}_{|y| \leq 1}\right) \nu_{\xi}(d y), \quad u \geq 0 .
\end{aligned}
$$

The formula given in Corollary 4.3 will be useful in determining $\mathcal{L}\left(\eta_{1}\right)$ from $\mathcal{L}\left(V_{\infty}\right)$ and $\mathcal{L}\left(\xi_{1}\right)$ as observed in Theorem 5.1 below. For the determination of $\mathcal{L}\left(\xi_{1}\right)$ from $\mathcal{L}\left(V_{\infty}\right)$ and $\mathcal{L}\left(\eta_{1}\right)$, the following relation between the characteristic triplets of $\xi, L$ and the characteristic function of $\log \left|V_{\infty}\right|$ will be helpful.

Theorem 4.6. Let $\left(\xi_{t}\right)_{t \geq 0}$ and $\left(\eta_{t}\right)_{t \geq 0}$ be two independent Lévy processes such that $V_{\infty}=$ $\int_{0}^{\infty} e^{-\xi_{s}-} d \eta_{s}$ converges to a finite random variable and such that $\eta$ is not the zero process. Then $\mu=\mathcal{L}\left(V_{\infty}\right)$ is the invariant law of the GOU process $\left(V_{t}\right)_{t \geq 0}$ driven by $(\xi, \eta)^{T}$.

Let $h \in C_{c}^{\infty}(\mathbb{R})$ such that $h(x)=1$ for $|x| \leq 1$ and $h(x)=0$ for $|x| \geq 2$ and set $h_{n}(x):=$ $h\left(\frac{x}{n}\right)$ and for $x \neq 0$ and $u \in \mathbb{R}$ define $f(x)=e^{i u \log |x|}$ and $f_{n}(x)=e^{i u \log |x|} h_{n}(\log |x|)$ with $f_{n}(0)=0$. Then

$$
\begin{aligned}
\psi_{-\xi}(u) \phi_{\log \left|V_{\infty}\right|}(u)= & -\lim _{n \rightarrow \infty} \int_{\mathbb{R}} A^{\eta} f_{n}(x) \mu(d x) \\
= & \lim _{n \rightarrow \infty}\left(-\gamma_{\eta} \int_{\mathbb{R}} f_{n}^{\prime}(x) \mu(d x)-\frac{\sigma_{\eta}^{2}}{2} \int_{\mathbb{R}} f_{n}^{\prime \prime}(x) \mu(d x)\right. \\
& -\int_{\mathbb{R}} \int_{\mathbb{R}}\left(f_{n}(x+y)-f_{n}(x)-f_{n}^{\prime}(x) y \mathbb{1}_{|y| \leq 1} \nu_{\eta}(d y) \mu(d x)\right) .
\end{aligned}
$$

If additionally $E\left[V_{\infty}^{-2}\right]<\infty$, then

$$
\begin{aligned}
\psi_{-\xi}(u) & \phi_{\log \left|V_{\infty}\right|}(u) \\
= & -i u \gamma_{\eta} E\left[V_{\infty}^{-1} e^{i u \log \left|V_{\infty}\right|}\right]+\frac{\sigma_{\eta}^{2}}{2}\left(i u+u^{2}\right) E\left[V_{\infty}^{-2} e^{i u \log \left|V_{\infty}\right|}\right] \\
& -\int_{\mathbb{R}}\left(E\left[e^{i u \log \left|V_{\infty}+y\right|}\right]-E\left[e^{i u \log \left|V_{\infty}\right|}\right]-i u y E\left[V_{\infty}^{-1} e^{i u \log \left|V_{\infty}\right|}\right] \mathbb{1}_{|y| \leq 1}\right) \nu_{\eta}(d y) .
\end{aligned}
$$

Proof. Observe that obviously $f_{n} \in C_{c}^{\infty}(\mathbb{R} ; \mathbb{C})$ and thus $f_{n} \in D\left(A^{V} ; \mathbb{C}\right) \cap D\left(A^{\eta} ; \mathbb{C}\right)$. On the other hand we obtain for $\tilde{f}(x)=f\left(e^{x}\right)$ and $\tilde{f}_{n}(x)=f_{n}\left(e^{x}\right)$ that $\tilde{f}(x)=e^{i u x}$ and $\tilde{f}_{n}(x)=$ $\tilde{f}(x) h_{n}(x)$ and hence $\tilde{f}_{n} \in C_{c}^{\infty}(\mathbb{R} ; \mathbb{C}) \subset D\left(A^{-\xi} ; \mathbb{C}\right)$. Similarly for $\tilde{\tilde{f}}(x)=f\left(-e^{x}\right)$ and $\tilde{\tilde{f}}_{n}(x)=f_{n}\left(-e^{x}\right)$ we have $\tilde{\tilde{f}}(x)=e^{i u x}$ and $\tilde{\tilde{f}}_{n}(x)=\tilde{\tilde{f}}(x) h_{n}(x)$ and also $\tilde{\tilde{f}}_{n} \in C_{c}^{\infty}(\mathbb{R} ; \mathbb{C}) \subset$ $D\left(A^{-\xi} ; \mathbb{C}\right)$.

Since $\mu(\{0\})=0$ by [6, Thm. 2.2], we obtain from (3.10) and (4.1)

$$
0=\int_{\mathbb{R}} A^{V} f_{n}(x) \mu(d x)
$$




$$
=\int_{\mathbb{R}} A^{\eta} f_{n}(x) \mu(d x)+\int_{(0, \infty)} A^{-\xi} \tilde{f}_{n}(\log x) \mu(d x)+\int_{(-\infty, 0)} A^{-\xi} \tilde{\tilde{f}}_{n}(\log |x|) \mu(d x) .
$$

Setting $S_{1}:(0, \infty) \rightarrow \mathbb{R}, x \mapsto \log x$, and $S_{2}:(-\infty, 0) \rightarrow \mathbb{R}, x \mapsto \log (-x)$, we compute using Lemma 4.2

$$
\begin{aligned}
& \lim _{n \rightarrow \infty}\left(\int_{(0, \infty)} A^{-\xi} \tilde{f}_{n}(\log x) \mu(d x)+\int_{(-\infty, 0)} A^{-\xi} \tilde{\tilde{f}}_{n}(\log |x|) \mu(d x)\right) \\
& =\lim _{n \rightarrow \infty}\left(\int_{\mathbb{R}} A^{-\xi} \tilde{f}_{n}(y) d S_{1}\left(\mu_{\mid(0, \infty)}\right)(y)+\int_{\mathbb{R}} A^{-\xi} \tilde{\tilde{f}}_{n}(y) d S_{2}\left(\mu_{\mid(-\infty, 0)}\right)(y)\right) \\
& =\psi_{-\xi}(u)\left(\int_{\mathbb{R}} e^{i u y} d S_{1}\left(\mu_{\mid(0, \infty)}\right)(y)+\int_{\mathbb{R}} e^{i u y} d S_{2}\left(\mu_{\mid(-\infty, 0)}\right)(y)\right) \\
& =\psi_{-\xi}(u)\left(\int_{(0, \infty)} e^{i u \log x} \mu(d x)+\int_{(-\infty, 0)} e^{i u \log |x|} \mu(d x)\right) \\
& =\psi_{-\xi}(u) \phi_{\log \left|V_{\infty}\right|}(u)
\end{aligned}
$$

which yields (4.9) and (4.10) via (3.1).

Now assume that $E\left[V_{\infty}^{-2}\right]<\infty$. We have $\tilde{f}_{n}(x)=e^{i u x} h_{n}(x)$ and $f_{n}(x)=\tilde{f}_{n}(\log |x|)$ for all $x \in \mathbb{R}$. In particular, $f_{n}^{\prime}(x)=x^{-1} \tilde{f}_{n}^{\prime}(\log |x|)$ and $f_{n}^{\prime \prime}(x)=x^{-2}\left(\tilde{f}_{n}^{\prime \prime}(\log |x|)-\tilde{f}_{n}^{\prime}(\log |x|)\right)$ for $x \neq 0$. For $|y|>1$, we further have $\left|f_{n}(x+y)-f_{n}(x)\right| \leq 2\|h\|<\infty$, and for $|y| \leq 1$ such that $x y(x+y) \neq 0$ there are $\zeta_{1}, \ldots, \zeta_{4} \in \mathbb{R}$ by Taylor's theorem such that

$$
\begin{aligned}
& \left|f_{n}(x+y)-f_{n}(x)-f_{n}^{\prime}(x) y\right| \\
& =\left|\tilde{f}_{n}(\log |x+y|)-\tilde{f}_{n}(\log |x|)-\tilde{f}_{n}^{\prime}(\log |x|) y x^{-1}\right| \\
& \leq\left|\tilde{f}_{n}\left((\log |x|)+y x^{-1}\right)-\tilde{f}_{n}(\log |x|)-\tilde{f}_{n}^{\prime}(\log |x|) y x^{-1}\right| \\
& +\left|\tilde{f}_{n}\left(\log |x|+\log \left|1+y x^{-1}\right|\right)-\tilde{f}_{n}\left((\log |x|)+y x^{-1}\right)\right| \\
& =2^{-1}\left|\left(\Re \tilde{f}_{n}^{\prime \prime}\right)\left((\log |x|)+\zeta_{1}\right) y^{2} x^{-2}\right|+\left|\left(\Re \tilde{f}_{n}^{\prime}\right)\left((\log |x|)+y x^{-1}+\zeta_{2}\right)\left(\log \left|1+y x^{-1}\right|-y x^{-1}\right)\right| \\
& +2^{-1}\left|\left(\Im \tilde{f}_{n}^{\prime \prime}\right)\left((\log |x|)+\zeta_{3}\right) y^{2} x^{-2}\right|+\left|\left(\Im \tilde{f}_{n}^{\prime}\right)\left((\log |x|)+y x^{-1}+\zeta_{4}\right)\left(\log \left|1+y x^{-1}\right|-y x^{-1}\right)\right| \\
& \leq\left\|\tilde{f}_{n}^{\prime \prime}\right\| y^{2} x^{-2}+\left\|\tilde{f}_{n}^{\prime}\right\| C y^{2} x^{-2}
\end{aligned}
$$

for some universal constant $C$. Since $\left\|\tilde{f}_{n}\right\|,\left\|\tilde{f}_{n}^{\prime}\right\|$ and $\left\|\tilde{f}_{n}^{\prime \prime}\right\|$ are uniformly bounded in $n$, since $\mu$ is continuous (cf. [6, Thm. 2]) so that $(\nu \otimes \mu)\left(\left\{(x, y)^{T} \in \mathbb{R}^{2}: x y(x+y)=0\right\}=0\right.$, since $\int_{\mathbb{R}} x^{-2} \mu(d x)<\infty$ by assumption and since $f_{n}, f_{n}^{\prime}$ and $f_{n}^{\prime \prime}$ converge on $\mathbb{R} \backslash\{0\}$ to $f$, $f^{\prime}$ and $f^{\prime \prime}$, respectively, by dominated convergence the right hand side of (4.10) is equal to

$$
\int_{\mathbb{R}}\left(-\gamma_{\eta} f^{\prime}(x)-\frac{\sigma_{\eta}^{2}}{2} f^{\prime \prime}(x)-\int_{\mathbb{R}}\left(f(x+y)-f(x)-f^{\prime}(x) y \mathbb{1}_{|y| \leq 1}\right) \nu_{\eta}(d y)\right) \mu(d x),
$$

which gives (4.11). 


\section{$5 \quad$ Injectivity}

Let $\xi=\left(\xi_{t}\right)_{t \geq 0}$ and $\left(\eta_{t}\right)_{t \geq 0}$ be two independent Lévy processes such that $V_{\infty}:=\int_{0}^{\infty} e^{-\xi_{s-}} d \eta_{s}$ converges almost surely. By [12, Thm. 2], this implies that $\xi$ drifts to $+\infty$. As in the introduction, for a Lévy process $\left(\xi_{t}\right)_{t \geq 0}$ such that $\xi_{t} \rightarrow+\infty$ a.s. as $t \rightarrow \infty$ denote

$D_{\xi}:=\left\{\mathcal{L}\left(\eta_{1}\right): \eta\right.$ Lévy process, independent of $\xi$, such that $\int_{0}^{\infty} e^{-\xi_{s-}} d \eta_{s}$ converges a.s. $\}$

and consider the mapping

$$
\Phi_{\xi}: D_{\xi} \rightarrow \mathcal{P}(\mathbb{R}), \quad \mathcal{L}\left(\eta_{1}\right) \mapsto \mathcal{L}\left(\int_{0}^{\infty} e^{-\xi_{s-}} d \eta_{s}\right), \quad \text { where } \eta \text { and } \xi \text { are independent. }
$$

Here $\mathcal{P}(\mathbb{R})$ denotes the set of probability distributions on $\left(\mathbb{R}, \mathcal{B}_{1}\right)$. For a Lévy process $\left(\eta_{t}\right)_{t \geq 0}$ denote further

$\tilde{D}_{\eta}:=\left\{\mathcal{L}\left(\xi_{1}\right): \xi\right.$ Lévy process, independent of $\eta$, such that $\int_{0}^{\infty} e^{-\xi_{s-}} d \eta_{s}$ converges a.s. $\}$

and define $\tilde{\Phi}_{\eta}$ by

$$
\tilde{\Phi}_{\eta}: \tilde{D}_{\eta} \rightarrow \mathcal{P}(\mathbb{R}), \quad \mathcal{L}\left(\xi_{1}\right) \mapsto \mathcal{L}\left(\int_{0}^{\infty} e^{-\xi_{s-}} d \eta_{s}\right), \quad \text { where } \eta \text { and } \xi \text { are independent. }
$$

We are interested in injectivity of the mappings $\Phi_{\xi}$ and $\tilde{\Phi}_{\eta}$, or at least in injectivity of these mappings when restricted to certain subsets. A key result for these investigations will be the following theorem, which follows immediately from (4.7) and (4.10), by dividing by $\phi_{V_{\infty}}(u)$ and $\phi_{\log \left|V_{\infty}\right|}(u)$ when different from zero, which is always the case for $u$ in a neighborhood of zero.

Theorem 5.1. Let $\left(\xi_{t}\right)_{t \geq 0}$ and $\left(\eta_{t}\right)_{t \geq 0}$ be two independent Lévy processes such that $V_{\infty}:=$ $\int_{0}^{\infty} e^{-\xi_{s-}} d \eta_{s}$ converges almost surely. If $\phi_{V_{\infty}}(u) \neq 0$ for $u$ from a dense subset of $\mathbb{R}$, or if $\mathcal{L}\left(\eta_{1}\right)$ is uniquely determined by the values of its characteristic function in a neighborhood of the origin, then $\mathcal{L}\left(\eta_{1}\right)$ is uniquely determined by $\mathcal{L}\left(V_{\infty}\right)$ and $\mathcal{L}\left(\xi_{1}\right)$. Similarly, if $\eta$ is not the zero process and $\phi_{\log \left|V_{\infty}\right|}(u) \neq 0$ for $u$ from a dense subset of $\mathbb{R}$, or if $\mathcal{L}\left(\xi_{1}\right)$ is uniquely determined by the values of its characteristic function in a neighborhood of the origin, then $\mathcal{L}\left(\xi_{1}\right)$ is uniquely determined by $\mathcal{L}\left(V_{\infty}\right)$ and $\mathcal{L}\left(\eta_{1}\right)$.

It is well known (e.g. [29]) that not every distribution is characterized by the values of its characteristic function in a neighborhood of the origin. This remains true for infinitely divisible distributions. To see this take two different distributions $\mu_{1}$ and $\mu_{2}$ without atoms at 0 whose characteristic functions coincide in a neighborhood of the origin and consider the corresponding compound Poisson distributions with Lévy measures $\mu_{1}$ and $\mu_{2}$. These are both infinitely divisible and their characteristic functions $\exp \left(\hat{\mu}_{i}(u)-1\right)$ coincide in a neighborhood of the origin. 
We do not know if the characteristic function of the stationary distribution of a GOU process cannot vanish on a non-empty open interval. As shown by Il'inskii [14, Cor. 1], a set $A \subset \mathbb{R}$ is the zero set of some characteristic function if and only if $A$ is closed, does not contain 0 and is symmetric with respect to the origin. Hence, a priori there is no reason why $\phi_{V_{\infty}}$ appearing in Theorem 5.1 should not vanish identically on some interval.

Still, it is possible to give some sufficient conditions. We start with the following lemma, which is a minor reformulation of results in Kawata [20] and Lucasz [30].

Lemma 5.2. Let $X$ be a random variable with law $\mu$ and assume that there is some $\varepsilon>0$ such that $E e^{\varepsilon X}<\infty$ or $E e^{-\varepsilon X}<\infty$. Then the characteristic function $\phi_{X}=\widehat{\mu}$ cannot be identically zero on non-empty open intervals. Furthermore, if $Y$ is another random variable whose characteristic function coincides with that of $X$ in a neighborhood of 0 , then $\mathcal{L}(Y)=\mathcal{L}(X)=\mu$.

Proof. Without loss of generality assume that $E e^{-\varepsilon X}<\infty$. Then $g(z):=E e^{i z X}$ can be defined for all $z \in \mathbb{C}$ such that $0 \leq \Im z<\varepsilon$, it is continuous there and analytic in $0<\Im z<\varepsilon$. That $\phi_{X}$ cannot be identically zero on non-empty open intervals then follows from [20, Cor. 1.14.1]. Let $Y$ be another random variable such that $\phi_{Y}(u)=\phi_{X}(u)$ for all $u \in(-a, a)$ with some $a>0$. Since $\phi_{Y}(u)=\lim _{y \downarrow 0} g(u+i y)$ for $u \in(-a, a)$, it follows from [30, Thm. 11.1.1] and its proof that $E e^{-\varepsilon Y}<\infty$. That $\mathcal{L}(Y)=\mathcal{L}(X)$ then follows from [20, Thm. 9.6.2].

Define $I D^{\text {sym }}$ to be the set of all infinitely divisible distributions $\mathcal{L}\left(\eta_{1}\right)$ which are symmetric, and $I D^{\exp }$ to be the set of all infinitely divisible distributions whose Lévy measure $\nu_{\eta}$ has some one-sided exponential moment, i.e. for which there is $\varepsilon>0$ such that

$$
\int_{1}^{\infty} e^{\varepsilon x} \nu_{\eta}(d x)<\infty \text { or } \quad \int_{-\infty}^{-1} e^{-\varepsilon x} \nu_{\eta}(d x)<\infty .
$$

Denote

$$
D_{\xi}^{\text {sym,exp }}:=D_{\xi} \cap\left(I D^{\text {sym }} \cup I D^{\text {exp }}\right), \quad D_{\xi}^{\text {sym }}:=D_{\xi} \cap I D^{\text {sym }} \quad \text { and } \quad D_{\xi}^{\text {exp }}:=D_{\xi} \cap I D^{\text {exp }} .
$$

With these notions, we get the following result:

Theorem 5.3. Let $\left(\xi_{t}\right)_{t \geq 0}$ be a Lévy process such that $\xi_{t}$ converges almost surely to $\infty$ as $t \rightarrow \infty$. Then $\left.\left(\Phi_{\xi}\right)\right|_{D_{\xi}^{\mathrm{sym}, \exp }}$ is injective and

$$
\Phi_{\xi}\left(D_{\xi}^{\text {sym,exp }}\right) \cap \Phi_{\xi}\left(D_{\xi} \backslash D_{\xi}^{\text {sym,exp }}\right)=\emptyset .
$$

If additionally $\xi$ is spectrally negative, or $\xi=q N$ for some constant $q>0$ and a Poisson process $N$, then $\Phi_{\xi}$ is injective on $D_{\xi}$.

In the special case when $\xi_{t}=t$, we have a spectrally negative $\xi$, and we recover the well known result (e.g. [16, Prop. 3.6.10]) that $\Phi_{\xi_{t}=t}$ is injective. 
Proof of Theorem 5.3. If $\xi$ is spectrally negative, then $V_{\infty}=\int_{0}^{\infty} e^{-\xi_{t-}} d \eta_{t}$ is self-decomposable by Remark (ii) to Theorem 2.2 in [6], hence infinitely divisible so that $\phi_{V_{\infty}}(u) \neq 0$ for all $u \in \mathbb{R}$. Injectivity of $\Phi_{\xi}$ then follows from Theorem 5.1. If $\xi=q N_{t}$ for $q>0$ and a Poisson process $N$, then by Example 1.1 we can write $V_{\infty}=\sum_{i=0}^{\infty} e^{-q i}\left(\eta_{T_{i+1}}-\eta_{T_{i}}\right)$, where $\left(\eta_{T_{i+1}}-\eta_{T_{i}}\right)_{i=0,1,2, \ldots}$ is i.i.d. and infinitely divisible by [36, Thm. 30.1]. Hence $V_{\infty}$ is infinitely divisible, and injectivity of $\Phi_{\xi}$ follows from Theorem 5.1 .

Now let $\xi$ be an arbitrary Lévy process drifting to infinity. If $\mathcal{L}\left(\eta_{1}\right) \in D_{\xi} \cap I D^{\exp }$, then there is $\varepsilon>0$ such that $E e^{\varepsilon \eta_{1}}<\infty$ or $E e^{-\varepsilon \eta_{1}}<\infty$ (cf. [36, Thm. 25.17]), and Theorem 5.1] and Lemma 5.2 show that $\left.\left(\Phi_{\xi}\right)\right|_{D_{\xi}^{\exp }}$ is injective and $\Phi_{\xi}\left(D_{\xi}^{\exp }\right) \cap \Phi_{\xi}\left(D_{\xi} \backslash D_{\xi}^{\exp }\right)=\emptyset$.

Finally, let $\xi$ be an arbitrary Lévy process drifting to infinity and $\mathcal{L}\left(\eta_{1}\right) \in D_{\xi}^{\text {sym }}$. Conditioning on $\xi$, for $f$ in the Skorokhod space $D([0, \infty), \mathbb{R})$ of càdlàg functions, we have

$$
\left(V_{\infty} \mid \xi=f\right)=\int_{0}^{\infty} e^{-f(t)} d \eta_{t}
$$

which converges for $P_{\xi^{-}}$almost every $f$. For such $f, \int_{0}^{\infty} e^{-f(t)} d \eta_{t}$ is infinitely divisible (e.g. Sato [37]), and hence $E\left(e^{i u V_{\infty}} \mid \xi=f\right) \neq 0$ for all $u \in \mathbb{R}$. Since $\int_{0}^{\infty} e^{-f(t)} d \eta_{t}$ is also symmetric, $E\left(e^{i u V_{\infty}} \mid \xi=f\right)$ is real valued and continuous in $u$ and hence strictly positive for all $u \in \mathbb{R}$. It follows that

$$
\phi_{V_{\infty}}(u)=\int_{D([0, \infty), \mathbb{R})} E\left[e^{i u V_{\infty}} \mid \xi=f\right] P_{\xi}(d f)>0 \quad \forall u \in \mathbb{R} .
$$

Theorem 5.1 then shows that $\left.\left(\Phi_{\xi}\right)\right|_{D_{\xi}^{\text {sym }}}$ is injective and $\Phi_{\xi}\left(D_{\xi}^{\text {sym }}\right) \cap \Phi_{\xi}\left(D_{\xi} \backslash D_{\xi}^{\text {sym }}\right)=\emptyset$. This finishes the proof.

Remark 5.4. Theorem 5.3 shows in particular that if $\xi$ is arbitrary (but drifting to $+\infty$ ), and $\eta$ is spectrally positive or negative (which applies in particular if $\eta$ is a subordinator or the negative of a subordinator), then the distribution of $\eta_{1}$ is uniquely determined by $\mathcal{L}\left(V_{\infty}\right)$ and $\mathcal{L}\left(\xi_{1}\right)$.

Let us now turn to injectivity properties of $\tilde{\Phi}_{\eta}$. We start with the following lemma, which is immediate from Lemma 5.2 .

Lemma 5.5. Let $X$ be a random variable which has no atom at 0 and assume that there is $\varepsilon>0$ such that $E|X|^{\varepsilon}<\infty$ or $E|X|^{-\varepsilon}<\infty$. Then the characteristic function $\phi_{\log |X|}$ of $\log |X|$ cannot be identically zero on non-empty open intervals.

Examples of random variables $X$ with finite negative fractional moment $E|X|^{-\varepsilon}<\infty$ are given by random variables which have a density $f$ in a neighborhood of zero such that $f(x)=O\left(x^{\alpha}\right)$ as $|x| \rightarrow 0$ for some $\alpha>\varepsilon-1$. In particular, if $\mathcal{L}(X)$ is a self-decomposable non-degenerate distribution, then $X$ has a density satisfying this condition for some $\varepsilon>0$, which follows from Theorems 28.4, 53.6 and 53.8 in [36]; observe that this is trivial if $X$ has a non-zero Gaussian component. Hence, whenever $X \not \equiv 0$ is self-decomposable, then $\phi_{\log |X|}$ cannot be identically zero on non-empty open intervals. 
Other examples are given in the next lemma, which shows that $\int_{0}^{\infty} e^{-\xi_{s-}} d \eta_{s}$ will always have certain negative fractional moments if $\eta$ is a subordinator with strictly positive drift, or if $\eta$ has a non-trivial Brownian motion component. This complements [31, Lem. 2.1] and [23, Lem. 3.3] who assume $\xi$ to have finite mean.

Lemma 5.6. Let $\xi$ and $\eta$ be two independent Lévy processes such that $V_{\infty}=\int_{0}^{\infty} e^{-\xi_{s-}} d \eta_{s}$ converges almost surely. Suppose that $\eta$ is a subordinator with strictly positive drift, or that the Brownian motion part of $\eta$ is non-trivial (i.e. $\sigma_{\eta}^{2}>0$ ). Then $E\left|V_{\infty}\right|^{-\varepsilon}<\infty$ for every $\varepsilon \in[0,1)$. In the latter case (i.e. when $\sigma_{\eta}^{2}>0$ ), $V_{\infty}$ has a bounded density on $\mathbb{R}$.

Proof. Suppose first that $\eta$ is a subordinator with strictly positive drift $\gamma_{\eta}^{0}$. Let $\varepsilon \in(0,1)$. Define the Lévy process $\xi^{b}$ by $\xi_{t}^{b}=\xi_{t}-\sum_{0<s<t,\left|\Delta \xi_{s}\right|>1} \Delta \xi_{s}$. Let $\tau$ be the time of the first jump of $\xi$ whose size is greater than 1 in magnitude. Then

$$
V_{\infty} \geq \gamma_{\eta}^{0} \int_{0}^{1 \wedge \tau} e^{-\xi_{s-}} d s \geq \gamma_{\eta}^{0}(1 \wedge \tau) \exp \left(-\sup _{0 \leq s \leq 1}\left|\xi_{s}^{b}\right|\right)
$$

Since $\tau$ and $\xi^{b}$ are independent and $\tau$ is exponentially distributed (or $\tau \equiv \infty$ ), it follows $E(1 \wedge \tau)^{-\varepsilon}<\infty$ and $E \exp \left(\varepsilon \sup _{0 \leq s \leq 1}\left|\xi_{s}^{b}\right|\right)<\infty$ (cf. [36, Thms. 25.17, 25.18]), so that $E V_{\infty}^{-\varepsilon}<\infty$ if $\eta$ is a subordinator with strictly positive drift.

Now suppose that $\eta$ is a Lévy process such that $\sigma_{\eta}^{2}>0$. Denote the Brownian motion component of $\eta$ by $B$, so that $B$ and $\eta-B$ are independent. Then the conditional distribution of $V_{\infty}$ given $\xi=f$ is given by $\int_{0}^{\infty} e^{-f(t-)} d B_{t}+\int_{0}^{\infty} e^{-f(t-)} d\left(\eta_{t}-B_{t}\right)$. But $\int_{0}^{\infty} e^{-f(t-)} d B_{t}$ is $N\left(0, \sigma_{\eta}^{2} \int_{0}^{\infty} e^{-2 f(s)} d s\right)$-distributed, hence its density is bounded by $\left(2 \pi \sigma_{\eta}^{2} \int_{0}^{\infty} e^{-2 f(s)} d s\right)^{-1 / 2}$. Hence also $\left(V_{\infty} \mid \xi=f\right)$ has a density, $g_{f}$ say, which is bounded by $\left(2 \pi \sigma_{\eta}^{2} \int_{0}^{\infty} e^{-2 f(s)} d s\right)^{-1 / 2}$. It follows that $V_{\infty}$ has a density given by $x \mapsto \int_{D([0, \infty))} g_{f}(x) P_{\xi}(d f)$, and since

$$
\int_{D([0, \infty))}\left(2 \pi \sigma_{\eta}^{2} \int_{0}^{\infty} e^{-2 f(s)} d s\right)^{-1 / 2} P_{\xi}(d f)=\left(2 \pi \sigma_{\eta}^{2}\right)^{-1 / 2} E\left[\left(\int_{0}^{\infty} e^{-2 \xi_{s-}} d s\right)^{-1 / 2}\right]<\infty
$$

by the part just proved, this density is bounded on $\mathbb{R}$. This then also shows that $E\left|V_{\infty}\right|^{-\varepsilon}<$ $\infty$ for all $\varepsilon \in[0,1)$.

Recall that $I D^{\exp }$ denotes the set of all infinitely divisible distributions whose Lévy measure has some one-sided exponential moment. Denote

$$
\tilde{D}_{\eta}^{\exp }:=\tilde{D}_{\eta} \cap I D^{\exp } \text {. }
$$

We can now prove the following injectivity result regarding $\tilde{\Phi}_{\eta}$ :

Theorem 5.7. Let $\eta=\left(\eta_{t}\right)_{t \geq 0}$ be a non-zero Lévy process. Then $\left.\left(\tilde{\Phi}_{\eta}\right)\right|_{\tilde{D}_{\eta}^{\exp }}$ is injective and

$$
\tilde{\Phi}_{\eta}\left(\tilde{D}_{\eta}^{\exp }\right) \cap \tilde{\Phi}_{\eta}\left(\tilde{D}_{\eta} \backslash \tilde{D}_{\eta}^{\exp }\right)=\emptyset .
$$

If additionally $\eta$ is a subordinator with strictly positive drift, or if the Brownian motion part of $\eta$ is non-trivial (i.e. $\sigma_{\eta}^{2}>0$ ), or if $\eta$ is a compound Poisson process without drift such that $\nu_{\eta}((-\infty, 0))=0$ and $\int_{0}^{1} x^{-\varepsilon} \nu_{\eta}(d x)<\infty$ for some $\varepsilon>0$, then $\tilde{\Phi}_{\eta}$ is injective on $\tilde{D}_{\eta}$. 
Observe that $\tilde{D}_{\eta}^{\text {exp }}$ contains all $\mathcal{L}\left(\xi_{1}\right) \in \tilde{D}_{\eta}$ such that $\xi$ is spectrally negative or spectrally positive. In particular, subordinators are uniquely determined by $\mathcal{L}\left(V_{\infty}\right)$ and $\mathcal{L}\left(\eta_{1}\right)$.

Proof of Theorem 5.7. The injectivity of $\tilde{\Phi}_{\eta}$ on $\tilde{D}_{\eta}^{\text {exp }}$ as well as (5.1) are clear from Theorem 5.1 and Lemma 5.2. Similarly, injectivity of $\tilde{\Phi}_{\eta}$ on $\tilde{D}_{\eta}$ follows from Lemmas 5.5, 5.6 and Theorem 5.1 if $\eta$ is a subordinator with strictly positive drift or if $\sigma_{\eta}^{2}>0$.

Finally, let us prove injectivity of $\tilde{\Phi}_{\eta}$ when $\eta$ is a compound Poisson process with $\nu_{\eta}((-\infty, 0))=$ 0 and $\int_{0}^{1} x^{-\varepsilon} \nu_{\eta}(d x)<\infty$ for some $\varepsilon>0$. Denote by $T$ the time of the first jump of $\eta$. Then

$$
V_{\infty}=\int_{0}^{\infty} e^{-\xi_{s-}} d \eta_{s}=e^{-\xi_{T-}} \Delta \eta_{T}+e^{-\xi_{T}} \int_{T}^{\infty} e^{-\left(\xi_{s-}-\xi_{T}\right)} d \eta_{s}=e^{-\xi_{T}}\left(\Delta \eta_{T}+V_{\infty}^{\prime}\right) \quad \text { a.s. },
$$

since $\xi$ and $\eta$ almost surely do not jump together. The random variable $V_{\infty}^{\prime}$ has the same distribution as $V_{\infty}$ and is independent of $\left(e^{-\xi_{T}}, \Delta \eta_{T}\right)$. Observe further that also $\xi_{T}$ and $\Delta \eta_{T}$ are independent. It follows that

$$
\phi_{\log V_{\infty}}(u)=\phi_{-\xi_{T}}(u) \phi_{\log \left(\Delta \eta_{T}+V_{\infty}^{\prime}\right)}(u), \quad u \in \mathbb{R} .
$$

Since

$$
E\left(\Delta \eta_{T}+V_{\infty}^{\prime}\right)^{-\varepsilon} \leq E\left(\Delta \eta_{T}\right)^{-\varepsilon}<\infty
$$

as a consequence of $V_{\infty}^{\prime} \geq 0$ and $\int_{0}^{1} x^{-\varepsilon} \nu_{\eta}(d x)<\infty$, it follows from Lemma 5.5 that $\phi_{\log \left(\Delta \eta_{T}+V_{\infty}^{\prime}\right)}$ cannot vanish identically on non-empty open intervals. Since $\phi_{-\xi_{T}}(u) \neq 0$ for all $u \in \mathbb{R}$ as $\xi_{T}$ is infinitely divisible, it follows that $\phi_{\log V_{\infty}}$ cannot vanish identically on non-empty open intervals. Injectivity of $\tilde{\Phi}_{\eta}$ then follows from Theorem 5.1.

We do not know if $\Phi_{\xi}$ and $\tilde{\Phi}_{\eta}$ will always be injective, but as we have seen in Theorems 5.3 and 5.7, the mappings $\Phi_{\xi}$ and $\tilde{\Phi}_{\eta}$ are injective in many cases. However, if we drop the condition of independence of $\xi$ and $\eta$, an injectivity result does not hold, as shown in the following. Therefore, additionally to the definitions at the beginning of this section, for a Lévy process $\xi$, let

$D_{\xi}^{\text {dep }}:=\left\{\mathcal{L}\left(\chi_{1}, \eta_{1}\right):(\chi, \eta)\right.$ biv. LP such that $\int_{0}^{\infty} e^{-\chi_{s-}} d \eta_{s}$ converges a.s. and $\left.\mathcal{L}\left(\chi_{1}\right)=\mathcal{L}\left(\xi_{1}\right)\right\}$ and define the mapping

$$
\Phi_{\xi}^{\mathrm{dep}}: D_{\xi}^{\mathrm{dep}} \rightarrow \mathcal{P}(\mathbb{R}), \quad \mathcal{L}\left(\chi_{1}, \eta_{1}\right) \mapsto \mathcal{L}\left(\int_{0}^{\infty} e^{-\chi_{s-}} d \eta_{s}\right)
$$

Then we obtain the following counterexample of injectivity.

Example 5.8. Let $\xi=N$ be a Poisson process. Then $\Phi_{\xi}^{\mathrm{dep}}$ is not injective.

Proof. Let $(\chi, \eta)$ be a bivariate Lévy process such that $\mathcal{L}\left(\chi_{1}, \eta_{1}\right) \in D_{\xi}^{\text {dep }}$. By [12, Thm. $2]$, this means $\mathcal{L}\left(\chi_{1}\right)=\mathcal{L}\left(\xi_{1}\right)$ and $E \log ^{+}\left|\eta_{1}\right|<\infty$. Denote the time of the first jump of $\chi$ by $T=T(\chi)$. Then

$$
\int_{0}^{\infty} e^{-\chi_{t-}} d \eta_{t}=\eta_{T}+e^{-1} \int_{T}^{\infty} e^{-\left(\chi_{t-}-\chi_{T}\right)} d \eta_{t}
$$


Since $\int_{T}^{\infty} e^{-\left(\chi_{t-}-\chi_{T}\right)} d \eta_{t}$ has the same distribution as $\int_{0}^{\infty} e^{-\chi_{t-}} d \eta_{t}=: W$, it follows that the characteristic function $\phi_{W}$ of $W$ satisfies

$$
\phi_{W}(x)=\prod_{k=0}^{\infty} \phi_{\eta_{T}}\left(e^{-k} x\right), \quad x \in \mathbb{R}
$$

as shown in [5]. Thus, $\mathcal{L}(W)$ is determined by $\rho_{\chi, \eta}:=\mathcal{L}\left(\eta_{T}\right)$ (not necessarily vice versa!). Now let $\left(\chi^{(1)}, \eta^{(1)}\right) \in D_{\xi}^{\text {dep }}$ be such that $\eta^{(1)}$ is independent of $\chi^{(1)}, \eta^{(1)}$ is not the zero process and $E \log ^{+}\left|\eta_{T\left(\chi^{(1)}\right)}^{(1)}\right|<\infty$, and let $\left(\chi^{(2)}, \eta^{(2)}\right)$ be a bivariate compound Poisson process without drift and Lévy measure

$$
\nu_{\chi^{(2)}, \eta^{(2)}}(d x, d y)=\delta_{1}(d x) \rho_{\chi^{(1)}, \eta^{(1)}}(d y) .
$$

Then $\left(\chi^{(2)}, \eta^{(2)}\right) \in D_{\xi}^{\text {dep }}$ and

$$
\rho_{\chi^{(2)}, \eta^{(2)}}=\mathcal{L}\left(\eta_{T\left(\chi^{(2)}\right)}^{(2)}\right)=\rho_{\chi^{(1)}, \eta^{(1)}} .
$$

It follows that both $\left(\chi^{(1)}, \eta^{(1)}\right)$ and $\left(\chi^{(2)}, \eta^{(2)}\right)$ lead to the same distribution, giving an example that injectivity is violated.

\section{$6 \quad$ Ranges}

The results of the previous section may now be used to determine information on the ranges of the mappings $\Phi_{\xi}$ and $\tilde{\Phi}_{\eta}$ as defined in Section 5. We start with an elementary conclusion, which also follows from [6, Thm. 2.2] or [3, Lem. 3.1].

Proposition 6.1. Let $\xi$ be non-deterministic, then $\Phi_{\xi}\left(D_{\xi} \backslash\{\mathcal{L}(0)\}\right)$ is a subset of the continuous distributions. Analoguously, if $\eta$ is non-deterministic, then the range of $\tilde{\Phi}_{\eta}$ is a subset of the continuous distributions.

Proof. It follows directly from [1, Thm. 1.3] that the distribution of the treated exponential functional fulfills a pure type theorem, in particular it is either continuous, or a Dirac measure. Suppose that $L_{1}=\eta_{1} \not \equiv 0$. Inserting the characteristic function $\phi(u)=e^{i u k}$, $k \in \mathbb{R}$, of a Dirac measure in (4.6), one immediately obtains $\psi_{L}(u)=-\psi_{U}(k u)$ which can only hold for deterministic processes $L_{t}=-k U_{t}=\gamma_{L} t$ with $k \neq 0$ and hence deterministic $\eta$ and $\xi$.

Recall the definition of $\Phi_{\xi}^{\text {dep }}$ from the previous section. Also recall that a distribution $\mu$ on $\left(\mathbb{R}, \mathcal{B}_{1}\right)$ is called $b$-decomposable, where $b \in(0,1)$, if there exists a probability measure $\rho$ on $\left(\mathbb{R}, \mathcal{B}_{1}\right)$ such that $\widehat{\mu}(z)=\widehat{\mu}(b z) \widehat{\rho}(z)$ for all $z \in \mathbb{R}$.

Proposition 6.2. Let $\xi=N$ be a Poisson process. Then the range of $\Phi_{\xi}^{\mathrm{dep}}$ is the class of all $e^{-1}$-decomposable distributions. 
Proof. That all distributions in the range of $\Phi_{\xi}^{\text {dep }}$ are $e^{-1}$-decomposable is clear from (5.2). Conversely, let $\mathcal{L}(W)$ be an $e^{-1}$-decomposable distribution. Then there exists an i.i.d. noise sequence $\left(Z_{n}\right)_{n \in \mathbb{N}_{0}}$ such that

$$
\sum_{k=0}^{n} e^{-k} Z_{k} \stackrel{d}{\rightarrow} W, \quad n \rightarrow \infty
$$

which follows by iterating the defining equation $W \stackrel{d}{=} e^{-1} W^{\prime}+Z$ with $W^{\prime}$ independent of $Z$ for $e^{-1}$-decomposability. Hence $\sum_{k=0}^{n} e^{-k} Z_{k}$ converges in distribution and hence almost surely as $n \rightarrow \infty$ and the Borel-Cantelli-lemma implies that $Z_{0}$ must have finite $\log ^{+}$moment. Now define the compound Poisson process $(\chi, \eta)$ without drift and Lévy measure

$$
\nu_{\chi, \eta}(d x, d y)=\delta_{1}(d x) \mathcal{L}\left(Z_{0}\right)(d y) .
$$

Then $\mathcal{L}(\chi, \eta) \in D_{\xi}^{\text {dep }}$ (due to the finite $\log ^{+}$moment of $Z_{0}$ ), and with the notations of Example 5.8 it follows that $\mathcal{L}\left(\eta_{T(\chi)}\right)=\mathcal{L}\left(Z_{0}\right)$. Hence $\Phi_{\xi}^{\mathrm{dep}}(\mathcal{L}(\chi, \eta))=\mathcal{L}(W)$.

Proposition 6.3. Let $\xi=N$ be a Poisson process. Then the range of $\Phi_{\xi}$ is a subset of the class of infinitely divisible $e^{-1}$-decomposable distributions without Gaussian part.

Proof. By Proposition 6.2 it remains to show that $W=\int_{(0, \infty)} e^{-N_{s-}} d \eta_{s}$ is infinitely divisible and has zero Gaussian part. Therefore denote the time of the first jump of $N$ by $T$, then $Z_{0}:=\eta_{T}$ is infinitely divisible without Gaussian part as a consequence of [36, Thm. 30.1]. Hence by (6.1) also $W$ is infinitely divisible and the Gaussian part of $W$ is zero.

It is well known that the OU process is a Gaussian process whose stationary distribution is normally distributed. In particular $\int_{(0, \infty)} e^{-t \sigma^{2} / 2} d\left(\sigma W_{t}\right)$ for $W_{t}$ a standard Brownian motion (Wiener process) is standard normally distributed. The following theorem shows that this is the only possible choice of $(\xi, \eta)^{T}$ which leads to a centered normal distribution.

Theorem 6.4. Let $\xi$ and $\eta$ be two independent Lévy processes such that $\int_{0}^{\infty} e^{-\xi_{s-}} d \eta_{s}$ converges almost surely. Let $v>0$. Then $\mathcal{L}\left(V_{\infty}\right)=N\left(0, v^{2}\right)$ if and only if there is $\gamma_{\xi}>0$ such that $\xi_{t}=\gamma_{\xi}$ t and $\eta_{t}=\left(2 \gamma_{\xi}\right)^{1 / 2} v W_{t}$, where $\left(W_{t}\right)_{t \geq 0}$ is a standard Brownian motion.

Proof. That $\mathcal{L}\left(V_{\infty}\right)=N\left(0, v^{2}\right)$ if $\xi$ and $\eta$ are as described is well known and follows as discussed above. Let us show the converse and assume that $V_{\infty}$ is $N\left(0, v^{2}\right)$-distributed. By replacing $\eta$ by $v^{-1} \eta$ we may assume that $v=1$. Inserting $\phi_{V_{\infty}}(u)=e^{-u^{2} / 2}$ in (4.8), we obtain for $u \in \mathbb{R}$

$$
\psi_{\eta}(u)=-\gamma_{\xi} u^{2}-\sigma_{\xi}^{2}\left(u^{4}-2 u^{2}\right) / 2-\int_{\mathbb{R}}\left(e^{-u^{2}\left(e^{-2 y}-1\right) / 2}-1-u^{2} y \mathbb{1}_{|y| \leq 1}\right) \nu_{\xi}(d y) .
$$

For given $u \in \mathbb{R}$ denote

$$
f_{u}(y):=e^{-u^{2}\left(e^{-2 y}-1\right) / 2}-1-u^{2} y \mathbb{1}_{|y| \leq 1}, \quad y \in \mathbb{R} \backslash\{0\} .
$$


We shall first investigate the limit behavior of (6.2) as $u \rightarrow \infty$ when divided by appropriate powers of $u$ and from that obtain information about the characteristic triplet of $\xi$. To do so, observe first that there are constants $C_{1}, C_{2}, C_{3}>0$ such that

$$
\begin{aligned}
\left|e^{-x}-1+x-x^{2} / 2\right| & \leq C_{1} x^{2} \quad \forall x>0, \\
\left|e^{-2 y}-1+2 y\right| & \leq C_{2} y^{2} \quad \forall y \in[-1,1], \quad \text { and } \\
\left(e^{-2 y}-1\right)^{2} & \leq C_{3} y^{2} \quad \forall y \in[-1,1] .
\end{aligned}
$$

Let $y_{0} \in[-1,0)$. Then $\left|f_{u}(y)\right| \leq 1+u^{2}$ for $y<y_{0}$, and for $y \in\left[y_{0}, 0\right)$ we can estimate

$$
\begin{aligned}
\left|f_{u}(y)\right| & \leq\left|-u^{2}\left(e^{-2 y}-1\right) / 2+u^{4}\left(e^{-2 y}-1\right)^{2} / 8-u^{2} y\right|+C_{1} u^{4}\left(e^{-2 y}-1\right)^{2} / 4 \\
& \leq u^{2} C_{2} y^{2} / 2+u^{4} C_{3} y^{2} / 8+C_{1} C_{3} u^{4} y^{2} / 4 .
\end{aligned}
$$

Using dominated convergence, this gives

$$
\limsup _{u \rightarrow \infty} u^{-4} \int_{-\infty}^{0}\left|f_{u}(y)\right| \nu_{\xi}(d y) \leq\left(C_{3} / 8+C_{1} C_{3} / 4\right) \int_{\left[y_{0}, 0\right)} y^{2} \nu_{\xi}(d y)
$$

and letting $y_{0} \uparrow 0$ we see that

$$
\lim _{u \rightarrow \infty} u^{-4} \int_{-\infty}^{0}\left|f_{u}(y)\right| \nu_{\xi}(d y)=0
$$

Now let $y>0$. Then

$$
f_{u}(y) \geq\left(u^{2}\left(1-e^{-2 y}\right) / 2-u^{2} y\right) \mathbb{1}_{(0,1]}(y) \geq-u^{2}\left(C_{2} / 2\right) y^{2} \mathbb{1}_{(0,1]}(y) .
$$

Since also $\lim _{u \rightarrow \infty} u^{-5} f_{u}(y)=+\infty$ for $y>0$ and $\lim _{u \rightarrow \infty} \int_{(0,1]} u^{-3} y^{2} \nu_{\xi}(d y)=0$, we obtain from Fatou's lemma

$$
\begin{aligned}
& \liminf _{u \rightarrow \infty} u^{-5} \int_{(0, \infty)} f_{u}(y) \nu_{\xi}(d y) \\
& \quad=\liminf _{u \rightarrow \infty} \int_{(0, \infty)} u^{-5}\left(f_{u}(y)+u^{2}\left(C_{2} / 2\right) y^{2} \mathbb{1}_{(0,1]}(y)\right) \nu_{\xi}(d y) \\
& \geq \int_{(0, \infty)} \liminf _{u \rightarrow \infty}\left(u^{-5} f_{u}(y)+u^{-3}\left(C_{2} / 2\right) y^{2} \mathbb{1}_{(0,1]}(y)\right) \nu_{\xi}(d y) \\
& =\int_{(0, \infty)} \infty \nu_{\xi}(d y)=\infty \nu_{\xi}((0, \infty)) .
\end{aligned}
$$

Dividing (6.2) by $u^{5}$ and observing that $\lim _{u \rightarrow \infty} u^{-2} \psi_{\eta}(u)=-\sigma_{\eta}^{2} / 2<\infty$ (cf. [36, Lem. 43.11]) and hence $\lim _{u \rightarrow \infty} u^{-5} \psi_{\eta}(u)=0$, this together with (6.3) gives $\nu_{\xi}((0, \infty))=0$. Similarly, dividing (6.2) by $u^{4}$, we obtain $\sigma_{\xi}^{2}=0$ by (6.3).

It remains to show that $\nu_{\xi}((-\infty, 0))=0$. In doing so, we shall first establish that $\xi$ must be of finite variation. Recall that

$$
e^{-x}-1+x \geq 0 \quad \forall x \geq 0 \text { and }
$$




$$
e^{-x}-1+x \geq x / 2 \quad \forall x \geq 4
$$

Let $y<0$. Then $f_{u}(y) \geq-1$ for $y<-1$, and for $y \in[-1,0)$ we estimate

$$
\begin{aligned}
f_{u}(y) & =e^{-u^{2}\left(e^{-2 y}-1\right) / 2}-1+u^{2}\left(e^{-2 y}-1\right) / 2-u^{2}\left(e^{-2 y}-1\right) / 2-u^{2} y \\
& \geq u^{2}\left(e^{-2 y}-1\right) / 4 \mathbb{1}_{\left\{u^{2}\left(e^{-2 y}-1\right) / 2 \geq 4\right\}}-C_{2} u^{2} y^{2} / 2 .
\end{aligned}
$$

An application of Fatou's lemma then shows

$$
\liminf _{u \rightarrow \infty} u^{-2} \int_{(-\infty, 0)} f_{u}(y) \nu_{\xi}(d y) \geq-C_{2} / 2 \int_{[-1,0)} y^{2} \nu_{\xi}(d y)+\int_{[-1,0)}\left(e^{-2 y}-1\right) / 4 \nu_{\xi}(d y) .
$$

But since $\lim _{u \rightarrow \infty} u^{-2}\left|\psi_{\eta}(u)\right|<\infty$, dividing (6.2) by $u^{2}$ and letting $u \rightarrow \infty$ gives $\int_{[-1,0)}\left(e^{-2 y}-\right.$ 1) $\nu_{\xi}(d y)<\infty$, hence $\int_{[-1,0)}|y| \nu_{\xi}(d y)<\infty$, so that $\xi$ is of finite variation. Equation (6.2) can now be rewritten as

$$
\psi_{\eta}(u)+\int_{(-\infty, 0)}\left(e^{-u^{2}\left(e^{-2 y}-1\right) / 2}-1\right) \nu_{\xi}(d y)=-\gamma_{\xi}^{0} u^{2},
$$

where $\gamma_{\xi}^{0}$ is the drift of $\xi$. Since $\xi_{t} \rightarrow \infty$ as $t \rightarrow \infty$ and $\xi$ is spectrally negative, we must have $\gamma_{\xi}^{0}>0$.

Let $\rho$ denote the standard normal distribution and define the mapping $T$ by

$$
T: \mathbb{R} \times(-\infty, 0) \rightarrow \mathbb{R}, \quad(x, y) \mapsto x \sqrt{e^{-2 y}-1} .
$$

Then for any $\varepsilon>0$,

$$
\begin{aligned}
& \int_{(-\infty,-\varepsilon]}\left(e^{-u^{2}\left(e^{-2 y}-1\right) / 2}-1\right) \nu_{\xi}(d y) \\
& =\left.\int_{\mathbb{R}} \int_{\mathbb{R}}\left(e^{i u x \sqrt{e^{-2 y}-1}}-1\right) \rho(d x) \nu_{\xi}\right|_{(-\infty,-\varepsilon]}(d y) \\
& =\int_{\mathbb{R}}\left(e^{i u z}-1\right) T\left(\left.\rho \otimes \nu_{\xi}\right|_{(-\infty,-\varepsilon]}\right)(d z) .
\end{aligned}
$$

With

$$
\bar{\psi}_{\varepsilon}(u):=\psi_{\eta}(u)+\int_{\mathbb{R}}\left(e^{i u z}-1\right) T\left(\left.\rho \otimes \nu_{\xi}\right|_{(-\infty,-\varepsilon]}\right)(d z)
$$

it follows from (6.4) that $\lim _{\varepsilon \downarrow 0} \bar{\psi}_{\varepsilon}(u)=-\gamma_{\xi}^{0} u^{2}$. But since $\bar{\psi}_{\varepsilon}$ is the Lévy-Khintchine exponent of an infinitely divisible distribution with Lévy measure $\nu_{\eta}+T\left(\left.\rho \otimes \nu_{\xi}\right|_{(-\infty,-\varepsilon]}\right)$, since $T\left(\left.\rho \otimes \nu_{\xi}\right|_{(-\infty,-\varepsilon]}\right)$ is increasing as $\varepsilon \downarrow 0$, and since $u \mapsto-\gamma_{\xi}^{0} u^{2}$ is the Lévy-Khintchine exponent of a Gaussian random variable, it follows from [36, Thm. 8.7] that $T\left(\left.\rho \otimes \nu_{\xi}\right|_{(-\infty,-\varepsilon]}\right)=$ 0 for any $\varepsilon>0$, hence $\nu_{\xi}((-\infty, 0))=0$.

We have shown that $\xi_{t}=\gamma_{\xi}^{0} t$. Injectivity of the mapping $\Phi_{\xi}$ (cf. Theorem 5.3) together with the sufficiency part show that necessarily $\eta_{t}=\left(2 \gamma_{\xi}\right)^{1 / 2} v W_{t}$, completing the proof. 


\section{Continuity}

Another natural question about the mappings $\Phi_{\xi}$ and $\tilde{\Phi}_{\eta}$ as defined in Section 5 is, whether they are continuous. Hereby we say, that $\Phi_{\xi}$ is continuous, if for each sequence of Lévy processes $\left(\eta^{(n)}\right)_{n \in \mathbb{N}}$ such that $\eta_{1}^{(n)} \stackrel{d}{\rightarrow} \eta_{1}$ as $n \rightarrow \infty$ and $\mathcal{L}\left(\eta_{1}^{(n)}\right) \in D_{\xi}, \mathcal{L}\left(\eta_{1}\right) \in D_{\xi}$, the sequence $\Phi_{\xi}\left(\mathcal{L}\left(\eta_{1}^{(n)}\right)\right)$ converges weakly to $\Phi_{\xi}\left(\mathcal{L}\left(\eta_{1}\right)\right)$ as $n \rightarrow \infty$, denoted as $\Phi_{\xi}\left(\mathcal{L}\left(\eta_{1}^{(n)}\right)\right) \stackrel{w}{\rightarrow}$ $\Phi_{\xi}\left(\mathcal{L}\left(\eta_{1}\right)\right)$ in the following. Continuity of $\tilde{\Phi}_{\eta}$ is defined similarly.

In general $\Phi_{\xi}$ is not continuous as proven by the following counterexample. We expect that failure of continuity of $\Phi_{\xi_{t}=t}$ is known as it is a very well studied mapping, but since we were unable to find a ready reference we give a short proof.

Example 7.1. Let $\left(\xi_{t}=t\right)_{t \geq 0}$ be deterministic. Then $\Phi_{\xi}$ is not continuous.

Proof. In the given setting we have that $D_{\xi}$ is $I D_{\log }$, the set of infinitely divisible distributions with finite $\log ^{+}$-moment. Now let $\left(Y_{i}^{(n)}\right)_{i \in \mathbb{N}}$ be sequences of i.i.d. random variables such that

$$
\nu^{(n)}:=\mathcal{L}\left(Y_{1}^{(n)}\right)=\left(1-\frac{1}{n}\right)\left(\frac{1}{2} \delta_{1}+\frac{1}{2} \delta_{-1}\right)+\frac{1}{n}\left(\frac{1}{2} \delta_{n^{n}}+\frac{1}{2} \delta_{-n^{n}}\right)
$$

and define the sequence $\left(Y_{i}^{(0)}\right)_{i \in \mathbb{N}}$ of i.i.d. random variables with

$$
\nu^{(0)}:=\mathcal{L}\left(Y_{1}^{(0)}\right)=\left(\frac{1}{2} \delta_{1}+\frac{1}{2} \delta_{-1}\right)
$$

Then obviously we have $Y_{i}^{(n)} \stackrel{d}{\rightarrow} Y_{i}^{(0)}$ as $n \rightarrow \infty$. Now for all $n \in \mathbb{N}_{0}$ define the compound Poisson process $\eta_{t}^{(n)}:=\sum_{i=1}^{N_{t}} Y_{i}^{(n)}$ where $N$ is a Poisson process with rate 1 , independent of $\left(Y_{i}^{(n)}\right)_{i \in \mathbb{N}}$. Then $\mu^{(n)}:=\mathcal{L}\left(\eta_{1}^{(n)}\right) \in D_{\xi}$ for all $n \in \mathbb{N}_{0}$ and in particular for $n \geq 1$ and $z \in \mathbb{R}$ we have that

$$
\begin{aligned}
& \widehat{\mu^{(n)}}(z)\left.=\exp \left(\int_{\mathbb{R}}\left(e^{i z x}-1\right) \nu^{(n)}(d x)\right)=\exp \widehat{\left(\nu^{(n)}\right.}(z)-1\right) \\
& \stackrel{n \rightarrow \infty}{\rightarrow} \exp \left(\widehat{\nu^{(0)}}(z)-1\right)=\widehat{\mu^{(0)}}(z)
\end{aligned}
$$

such that $\mu^{(n)} \rightarrow \mu^{(0)}$ as $n \rightarrow \infty$. But $\phi_{\xi}\left(\mu^{(n)}\right)$ does not converge to $\phi_{\xi}\left(\mu^{(0)}\right)$ as will be shown in the following. Herefore observe that by [36, Eq. (17.14)] the Lévy measure $\tilde{\nu}^{(n)}$ of $\phi_{\xi}\left(\mu^{(n)}\right)$ fulfills for all $n \geq 0$

$$
\tilde{\nu}^{(n)}([1, \infty))=\int_{\mathbb{R}} \int_{0}^{\infty} \mathbb{1}_{[1, \infty)}\left(e^{-s} y\right) d s \nu^{(n)}(d y)=\int_{(0, \infty)} \log y \nu^{(n)}(d y)
$$

such that for all $n \geq 1$

$$
\tilde{\nu}^{(n)}([1, \infty))=\frac{1}{2} \log n \rightarrow \infty \text { as } n \rightarrow \infty
$$

whereas $\tilde{\nu}^{(0)}([1, \infty))=0$. Using [36, Thm. 8.7] this shows that $\Phi_{\xi}\left(\mu^{(n)}\right) \stackrel{\not p}{\rightarrow} \Phi_{\xi}(\mu)$ as $n \rightarrow \infty$, so that $\Phi_{\xi}$ is not continuous. 
Continuity of stationary solutions of random recurrence equations has been studied by Brandt [8]. The following is a special case of his result for i.i.d. sequences, but does not assume that $E\left[\log \left|B_{0}^{(n)}\right|\right], E\left[\left|\log B_{0}\right|\right]$ are finite and that $E\left[\log \left|B_{0}^{(n)}\right|\right] \rightarrow E\left[|\log | B_{0} \mid\right]$ as $n \rightarrow \infty$. That these conditions can be omitted follows readily by an inspection of Brandt's proof [8, Thm. 2].

Proposition 7.2. Let the sequences $\left(A_{i}, B_{i}\right)_{i \in \mathbb{N}_{0}},\left(A_{i}^{(1)}, B_{i}^{(1)}\right)_{i \in \mathbb{N}_{0}}\left(A_{i}^{(2)}, B_{i}^{(2)}\right)_{i \in \mathbb{N}_{0}}, \ldots$ be i.i.d. such that $E\left[\log ^{+}\left|A_{0}^{(n)}\right|\right]<\infty, E\left[\log ^{+}\left|B_{0}^{(n)}\right|\right]<\infty$ for all $n, E\left[\log ^{+}\left|A_{0}\right|\right]<\infty$ and $E\left[\log ^{+}\left|B_{0}\right|\right]<\infty$. Assume further that

$$
-\infty<E\left[\log \left|A_{0}^{(n)}\right|\right]<0 \quad \text { for all } n, \quad-\infty<E\left[\log \left|A_{0}\right|\right]<0
$$

and that for $n \rightarrow \infty$

$$
\begin{aligned}
\left(A_{0}^{(n)}, B_{0}^{(n)}\right) & \stackrel{d}{\rightarrow}\left(A_{0}, B_{0}\right), \\
E\left[\log ^{+}\left|A_{0}^{(n)}\right|\right] & \rightarrow E\left[\log ^{+}\left|A_{0}\right|\right], \\
E\left[\log ^{+}\left|B_{0}^{(n)}\right|\right] & \rightarrow E\left[\log ^{+}\left|B_{0}\right|\right] \\
\text { and } E\left[\log \left|A_{0}^{(n)}\right|\right] & \rightarrow E\left[\log \left|A_{0}\right|\right] .
\end{aligned}
$$

Let $Y_{\infty}^{(n)}$ be the unique stationary marginal distribution of the random recurrence equation $Y_{i+1}^{(n)}=A_{i}^{(n)} Y_{i}^{(n)}+B_{i}^{(n)}, i \in \mathbb{N}_{0}$, and define $Y_{\infty}$ analoguously. Then

$$
\left(A_{0}^{(n)}, B_{0}^{(n)}, Y_{\infty}^{(n)}\right) \stackrel{d}{\rightarrow}\left(A_{0}, B_{0}, Y_{\infty}\right) \quad \text { as } n \rightarrow \infty
$$

such that in particular $Y_{\infty}^{(n)} \stackrel{d}{\rightarrow} Y_{\infty}$ as $n \rightarrow \infty$.

Due to the fact that generalized Ornstein-Uhlenbeck processes are the continuous-time analogon of the solutions to random recurrence equations with i.i.d. coefficients, we can use the above proposition in our setting to obtain the following.

Theorem 7.3. Let $\left(\xi^{(n)}, \eta^{(n)}\right), n \in \mathbb{N}$, and $(\xi, \eta)$ be bivariate Lévy processes such that

$$
\left(\xi_{1}^{(n)}, \eta_{1}^{(n)}\right) \stackrel{d}{\rightarrow}\left(\xi_{1}, \eta_{1}\right), \quad n \rightarrow \infty .
$$

Suppose there exists $\delta>0$ such that

$$
\begin{aligned}
& \sup _{n \in \mathbb{N}} \int_{\mathbb{R} \backslash[-1,1]}\left(\log ^{+}|x|\right)^{1+\delta} \nu_{\eta^{(n)}}(d x)<\infty \\
& \text { and } \sup _{n \in \mathbb{N}} E\left[\left|\xi_{1}^{(n)}\right|^{1+\delta}\right]<\infty .
\end{aligned}
$$

Then $E \log ^{+}\left|\eta_{1}\right|<\infty$ and $E\left|\xi_{1}\right|<\infty$. Assume further that

$$
E \xi_{1}>0 \text { and } E \xi_{1}^{(n)}>0, n \in \mathbb{N} .
$$

Then $\int_{0}^{\infty} e^{-\xi_{s-}^{(n)}} d \eta_{s}^{(n)}$ converges almost surely absolutely for each $n \in \mathbb{N}$, as does $\int_{0}^{\infty} e^{-\xi_{s-}} d \eta_{s}$, and

$$
\int_{0}^{\infty} e^{-\xi_{s-}^{(n)}} d \eta_{s}^{(n)} \stackrel{d}{\rightarrow} \int_{0}^{\infty} e^{-\xi_{s-}} d \eta_{s}, \quad n \rightarrow \infty .
$$


For the proof of Theorem 7.3 we need the following Lemma, which is of its own interest.

Lemma 7.4. Let $L=\left(L_{t}\right)_{t \geq 0}$ be a Lévy process in $\mathbb{R}$ with characteristic triplet $\left(\gamma_{L}, \sigma_{L}^{2}, \nu_{L}\right)$. Let $b>0$. Then there exist universal constants $C_{1}, C_{2}, C_{3} \in(0, \infty)$, depending only on $b$, such that for every adapted càdlàg process $H$ satisfying

$$
E\left(\log ^{+} \sup _{0 \leq s \leq 1}\left|H_{s}\right|\right)^{b}<\infty
$$

the following estimate holds:

$$
\begin{aligned}
& E\left(\log ^{+} \sup _{0<s \leq 1}\left|\int_{0}^{s} H_{u-} d L_{u}\right|\right)^{b} \\
& \leq C_{1}\left(1+\sigma_{L}^{2}+\int_{|x| \leq 1} x^{2} \nu_{L}(d x)+\log ^{+}\left|\gamma_{L}\right|+\exp \left\{C_{2} \int_{|x|>1}\left(\log ^{+}|x|\right)^{b} \nu_{L}(d x)\right\}\right) \\
& \quad+C_{3} E\left(\log ^{+} \sup _{0 \leq s \leq 1}\left|H_{s}\right|\right)^{b} .
\end{aligned}
$$

Proof. Write $L_{t}=L_{t}^{\sharp}+L_{t}^{b}$, where $L^{\sharp}=\left(L_{t}^{\sharp}\right)_{t \geq 0}$ has characteristic triplet

$$
\left(\gamma_{L}^{\sharp}:=0,\left(\sigma_{L}^{\sharp}\right)^{2}:=\sigma_{L}^{2}, \nu_{L}^{\sharp}:=\left.\nu_{L}\right|_{[-1,1]}\right)
$$

and $L^{b}=\left(L_{t}^{b}\right)_{t \geq 0}$ has characteristic triplet

$$
\left(\gamma_{L}^{b}:=\gamma_{L},\left(\sigma_{L}^{b}\right)^{2}:=0, \nu_{L}^{b}:=\left.\nu_{L}\right|_{\mathbb{R} \backslash[-1,1]}\right)
$$

Then $L^{\sharp}$ has expectation zero (e.g. [36, Ex. 25.12]) and is a square integrable martingale, and $L^{b}$ is a compound Poisson process together with drift $\gamma_{L}$. Observe that for proving (7.5) it is obviously sufficient to prove it for $L^{\sharp}$ and $L^{b}$ separately, which we shall do.

For the estimate for $L^{\sharp}$, let $x>0$. Then

$$
\begin{aligned}
P & \left(\left(\log ^{+} \sup _{0<s \leq 1}\left|\int_{0}^{s} H_{u-} d L_{u}^{\sharp}\right|\right)^{b}>x\right) \\
= & P\left(\sup _{0<s \leq 1}\left|\int_{0}^{s} H_{u-} d L_{u}^{\sharp}\right|>\exp \left(x^{1 / b}\right)\right) \\
\leq & P\left(\sup _{0<s \leq 1}\left|\int_{0}^{s} H_{u-} d L_{u}^{\sharp}\right|>\exp \left(x^{1 / b}\right), \sup _{0 \leq s \leq 1}\left|H_{s}\right| \leq \exp \left(x^{1 / b} / 2\right)\right) \\
& +P\left(\sup _{0 \leq s \leq 1}\left|H_{s}\right|>\exp \left(x^{1 / b} / 2\right)\right) .
\end{aligned}
$$

Denote $H_{s}^{(x)}:=H_{s} \wedge \exp \left(x^{1 / b} / 2\right)$. Then on $\left\{\sup _{0 \leq s \leq 1}\left|H_{s}\right| \leq \exp \left(x^{1 / b} / 2\right)\right\}, \int_{0}^{s} H_{u-} d L_{u}^{\sharp}=$ $\int_{0}^{s} H_{u-}^{(x)} d L_{u}^{\sharp}$ for all $0 \leq s \leq 1$, so that by Markov's inequality and Doob's maximal quadratic inequality, we obtain

$$
P\left(\sup _{0<s \leq 1}\left|\int_{0}^{s} H_{u-} d L_{u}^{\sharp}\right|>\exp \left(x^{1 / b}\right), \sup _{0 \leq s \leq 1}\left|H_{s}\right| \leq \exp \left(x^{1 / b} / 2\right)\right)
$$




$$
\begin{aligned}
& \leq P\left(\sup _{0 \leq s \leq 1}\left|\int_{0}^{1} H_{u-}^{(x)} d L_{u}^{\sharp}\right|>\exp \left(x^{1 / b}\right)\right) \\
& \leq \exp \left(-2 x^{1 / b}\right) E \sup _{0 \leq s \leq 1}\left|\int_{0}^{1} H_{u-}^{(x)} d L_{u}^{\sharp}\right|^{2} \\
& \leq 4 \exp \left(-2 x^{1 / b}\right) E\left|\int_{0}^{1} H_{u-}^{(x)} d L_{u}^{\sharp}\right|^{2} \\
& =4 \exp \left(-2 x^{1 / b}\right) \int_{0}^{1} E\left|H_{u-}^{(x)}\right|^{2} d L_{u}^{\sharp} \operatorname{Var}\left(L_{1}^{\sharp}\right) \\
& \leq 4 \exp \left(-2 x^{1 / b}\right) \exp \left(x^{1 / b}\right) \operatorname{Var}\left(L_{1}^{\sharp}\right) \\
& =4 \exp \left(-x^{1 / b}\right)\left(\sigma_{L}^{2}+\int_{|y| \leq 1} y^{2} \nu_{L}(d y)\right),
\end{aligned}
$$

where we used [36, Ex. 25.12] to express the variance $\operatorname{Var}\left(L_{1}^{\sharp}\right)$ in terms of the characteristic triplet. Combining this with (7.6), we obtain

$$
\begin{aligned}
& E\left(\log ^{+} \sup _{0<s \leq 1}\left|\int_{0}^{s} H_{u-} d L_{u}^{\sharp}\right|\right)^{b} \\
& \leq 4\left(\sigma_{L}^{2}+\int_{|y| \leq 1} y^{2} \nu_{L}(d y)\right) \int_{0}^{\infty} \exp \left(-x^{1 / b}\right) d x+\int_{0}^{\infty} P\left(\left(\log ^{+} \sup _{0 \leq s \leq 1}\left|H_{s}\right|\right)^{b}>x 2^{-b}\right) d x \\
& =4\left(\sigma_{L}^{2}+\int_{|y| \leq 1} y^{2} \nu_{L}(d y)\right) \int_{0}^{\infty} \exp \left(-x^{1 / b}\right) d x+2^{b} E\left(\log ^{+} \sup _{0 \leq s \leq 1}\left|H_{s}\right|\right)^{b},
\end{aligned}
$$

establishing (7.5) for $L^{\sharp}$.

In order to obtain (7.5) for $L^{b}$, denote

$$
R_{t}:=\left|\gamma_{L}\right| t+\sum_{0<s \leq t}\left|\Delta L_{s}^{b}\right|
$$

Then $R=\left(R_{t}\right)_{t \geq 0}$ is a subordinator and

$$
\begin{aligned}
& \left(\log ^{+} \sup _{0 \leq s \leq 1}\left|\int_{0}^{s} H_{u-} d L_{u}^{b}\right|\right)^{b} \\
& \leq\left(\log ^{+}\left(R_{1} \sup _{0 \leq s \leq 1}\left|H_{s}\right|\right)\right)^{b} \\
& \leq\left(\log ^{+} R_{1}+\log ^{+} \sup _{0<s \leq 1}\left|H_{s}\right|\right)^{b} \\
& \leq\left(2^{b-1} \vee 1\right)\left(\log ^{+} R_{1}\right)^{b}+\left(2^{b-1} \vee 1\right)\left(\log ^{+} \sup _{0 \leq s \leq 1}\left|H_{s}\right|\right)^{b} .
\end{aligned}
$$

Since the function $x \mapsto(\log (x \vee e))^{b}$ is submultiplicative (cf. Sato [36, Prop. 25.4]), it follows from the proof of Theorem 25.3 in Sato [36] that there is a constant $C_{2}=C_{2}(b)$, 
depending only on $b$, such that

$$
E\left(\log \left(e \vee \sum_{0<s \leq 1}\left|\Delta L_{s}^{b}\right|\right)\right)^{b} \leq \exp \left\{C_{2} \int_{|x|>1}\left(\log ^{+}|x|\right)^{b} \nu_{L}(d x)\right\} .
$$

Hence, there is a constant $C_{4}=C_{4}(b) \in(0, \infty)$ such that

$$
\begin{aligned}
& E\left(\log ^{+} R_{1}\right)^{b} \\
& \leq 1+E\left(\log \left(e \vee R_{1}\right)\right)^{b} \\
& \leq C_{4}\left(1+\left(\log ^{+}\left|\gamma_{L}\right|\right)^{b}+\exp \left\{C_{2} \int_{|x|>1}\left(\log ^{+}|x|\right)^{b} \nu_{L}(d x)\right\}\right) .
\end{aligned}
$$

Together with (7.7) this gives (7.5) for $L^{b}$.

Proof of Theorem 7.3. Recall that for any real numbers $a$ and $b$ and $\delta>0$ it holds

$$
|a+b|^{1+\delta} \leq C_{\delta}\left(|a|^{1+\delta}+|b|^{1+\delta}\right)
$$

for some constant $C_{\delta}$. Using this together with Doob's martingale inequality (c.f. [36, Eq. (25.16)]) and Jensen's inequality we obtain

$$
\begin{aligned}
E\left[\sup _{0<s \leq 1}\left|\xi_{s}^{(n)}\right|^{1+\delta}\right] & \leq C_{\delta}\left(E\left[\sup _{0<s \leq 1}\left|\xi_{s}^{(n)}-s E\left[\xi_{1}^{(n)}\right]\right|^{1+\delta}\right]+\left|E\left[\xi_{1}^{(n)}\right]\right|^{1+\delta}\right) \\
& \leq C_{\delta}\left(8 E\left[\left|\xi_{1}^{(n)}-E\left[\xi_{1}^{(n)}\right]\right|^{1+\delta}\right]+\left|E\left[\xi_{1}^{(n)}\right]\right|^{1+\delta}\right) \\
& \leq 8 C_{\delta}^{2} E\left[\left|\xi_{1}^{(n)}\right|^{1+\delta}\right]+\left(8 C_{\delta}^{2}+C_{\delta}\right)\left|E\left[\xi_{1}^{(n)}\right]\right|^{1+\delta} \\
& \leq\left(16 C_{\delta}^{2}+C_{\delta}\right) E\left[\left|\xi_{1}^{(n)}\right|^{1+\delta}\right] .
\end{aligned}
$$

Hence from (7.2) we conclude

$$
\sup _{n \in \mathbb{N}} E\left[\left(\sup _{0<s \leq 1}\left|\xi_{s}^{(n)}\right|\right)^{1+\delta}\right]<\infty
$$

and therefore also

$$
\sup _{n \in \mathbb{N}} E\left[\left(\sup _{0<s \leq 1}\left|\xi_{s}^{(n)} \vee 0\right|\right)^{1+\delta}\right]<\infty .
$$

Denote by $\left(\gamma_{\eta^{(n)}}, \sigma_{\eta^{(n)}}^{2}, \nu_{\eta^{(n)}}\right)$ and $\left(\gamma_{\eta}, \sigma_{\eta}^{2}, \nu_{\eta}\right)$ the characteristic triplets of $\eta^{(n)}$ and $\eta$, respectively. Denote by $h$ the continuous truncation function $h(x)=x \mathbf{1}_{|x| \leq 1}+(2-|x|) \operatorname{sgn}(x) \mathbf{1}_{|x| \in(1,2]}$. Set

$\beta_{\eta^{(n)}}:=\gamma_{\eta^{(n)}}+\int_{[-2,2]}\left(h(x)-x \mathbf{1}_{|x| \leq 1}\right) \nu_{\eta^{(n)}}(d x)=\gamma_{\eta^{(n)}}+\int_{[-2,2]} x\left(\frac{h(x)}{x}-\mathbf{1}_{|x| \leq 1}\right) \nu_{\eta^{(n)}}(d x)$,

i.e. the constant term in the Lévy-Khintchine triplet of $\eta^{(n)}$ with respect to the truncation function $h$ (c.f. [36, Eqs. (8.5), (8.6)]). Define $\beta_{\eta}$ similarly. Since $\eta_{1}^{(n)} \stackrel{d}{\rightarrow} \eta_{1}$, it follows from [15, Thm. VII.2.9, p.396] that $\beta_{\eta^{(n)}} \rightarrow \beta_{\eta}$,

$$
\sigma_{\eta^{(n)}}^{2}+\int_{|x| \leq 1} x^{2} \nu_{\eta^{(n)}}(d x)+\int_{1<|x| \leq 2}(2-|x|)^{2} \nu_{\eta^{(n)}}(d x)
$$




$$
\rightarrow \sigma_{\eta}^{2}+\int_{|x| \leq 1} x^{2} \nu_{\eta}(d x)+\int_{1<|x| \leq 2}(2-|x|)^{2} \nu_{\eta}(d x)
$$

and $\int_{\mathbb{R}} f(x) \nu_{\xi^{(n)}}(d x) \rightarrow \int_{\mathbb{R}} f(x) \nu_{\xi}(d x)$ as $n \rightarrow \infty$ for every continuous bounded function $f$ vanishing in a neighbourhood of zero. In particular,

$$
\sup _{n \in \mathbb{N}} \sigma_{\eta^{(n)}}^{2}<\infty, \quad \sup _{n \in \mathbb{N}} \int_{[-1,1]} x^{2} \nu_{\eta^{(n)}}(d x)<\infty \quad \text { and } \quad \sup _{n \in \mathbb{N}}\left|\gamma_{\eta^{(n)}}\right|<\infty .
$$

Applying Lemma 7.4 with $b=1+\delta$ and $H_{s}=1$ and using (7.1) then shows that $\sup _{n \in \mathbb{N}} E\left(\log ^{+}\left|\eta_{1}^{(n)}\right|\right)^{1+\delta}<\infty$, and hence that $E\left(\log ^{+}\left|\eta_{1}\right|\right)^{1+\delta}<\infty$ by Fatou's lemma for weak convergence (cf. Kallenberg [17, Lem. 4.11]), and similarly we obtain $E\left|\xi_{1}\right|^{1+\delta}<\infty$ from (7.8).

Since $E \log ^{+}\left|\eta_{1}\right|<\infty, E \xi_{1}>0, E \log ^{+}\left|\eta_{1}^{(n)}\right|<\infty$ and $E \xi_{1}^{(n)}>0$, the integrals $\int_{0}^{\infty} e^{-\xi_{s-}} d \eta_{s}$ and $\int_{0}^{\infty} e^{-\xi_{s-}^{(n)}} d \eta_{s}^{(n)}$ converge almost surely absolutely (cf. [12, Thm. 2]). Writing

$$
\int_{0}^{\infty} e^{-\xi_{s-}^{(n)}} d \eta_{s}^{(n)}=\int_{0}^{1} e^{-\xi_{s-}^{(n)}} d \eta_{s}^{(n)}+e^{-\xi_{1}^{(n)}} \int_{1}^{\infty} e^{-\left(\xi_{s-}^{(n)}-\xi_{1}^{(n)}\right)} d\left(\eta_{s}^{(n)}-\eta_{1}^{(n)}\right),
$$

we have

$$
\int_{0}^{\infty} e^{-\xi_{s-}^{(n)}} d \eta_{s}^{(n)} \stackrel{d}{=} \sum_{k=0}^{\infty}\left(\prod_{i=0}^{k-1} A_{i}^{(n)}\right) B_{k}^{(n)}
$$

with some i.i.d. sequences $\left(A_{k}^{(n)}, B_{k}^{(n)}\right)_{k \in \mathbb{N}_{0}}$ such that

$$
\left(A_{0}^{(n)}, B_{0}^{(n)}\right)=\left(e^{-\xi_{1}^{(n)}}, \int_{0}^{1} e^{-\xi_{s-}^{(n)}} d \eta_{s}^{(n)}\right)
$$

and a similar statement holds for $\int_{0}^{\infty} e^{-\xi_{s-}} d \eta_{s}$ with $\left(A_{k}, B_{k}\right)_{k \in \mathbb{N}_{0}}$ i.i.d. such that $\left(A_{0}, B_{0}\right)=$ $\left(e^{-\xi_{1}}, \int_{0}^{1} e^{-\xi_{s-}} d \eta_{s}\right)$.

Now, to apply Proposition 7.2, we have to check its conditions on the sequences $\left(A_{0}^{(n)}\right)_{n \in \mathbb{N}}$ and $\left(B_{0}^{(n)}\right)_{n \in \mathbb{N}}$ which we shall do in the following.

Since $\left(\xi_{1}^{(n)}, \eta_{1}^{(n)}\right) \stackrel{d}{\rightarrow}\left(\xi_{1}, \eta_{1}\right), n \rightarrow \infty$, it follows from [15, Cor. VII.3.6, p. 415] that $\left(\xi^{(n)}, \eta^{(n)}\right) \stackrel{\mathcal{L}}{\rightarrow}(\xi, \eta)$, where $\stackrel{\mathcal{L}}{\rightarrow}$ " denotes convergence in the Skorokhod topology. Additionally, the sequences $\left(\xi^{(n)}\right), n \in \mathbb{N}$, and $\left(\eta^{(n)}\right), n \in \mathbb{N}$, satisfy the P-UT condition (cf. [15, Def. VI.6.1, p. 377]). To see this, let $h: \mathbb{R}^{2} \rightarrow \mathbb{R}^{2}$ be a continuous bounded function satisfying $h(x)=x$ in a neighbourhood of 0 . Then if $\left(\gamma_{h}, A, \nu\right)_{h}$ is the Lévy-Khintchine triplet of $(\xi, \eta)$ with respect to $h$ (we use the notations here as in [15, Eq. II.4.21, p. 107]), then $\left(\gamma_{h} t, A t, d t \nu(d x)\right), t \geq 0$, is the semimartingale characteristic of $(\xi, \eta)$ with respect to $h$, cf. [15, Cor. II.4.19, p. 107]. A similar statement holds for $\left(\xi^{(n)}, \eta^{(n)}\right)$. Since $\left(\xi^{(n)}, \eta^{(n)}\right) \stackrel{\mathcal{L}}{\rightarrow}(\xi, \eta)$ as $n \rightarrow \infty$, the sequence $\left(\xi^{(n)}, \eta^{(n)}\right), n \in \mathbb{N}$, is tight. Furthermore, since again by [15, Cor. VII.3.6, p. 415], $\gamma_{h}^{(n)} \rightarrow \gamma_{h}$ as $n \rightarrow \infty$, and since the total variation of $s \mapsto \gamma_{h}^{(n)} s$ on $[0, t]$ is $\left|\gamma_{h}^{(n)}\right| t$, condition (iii) of [15, Thm. VI.6.15, p. 380] is satisfied, and it follows from [15, Thm. VI.6.21, p. 382] that $\left(\xi^{(n)}, \eta^{(n)}\right), n \in \mathbb{N}$, is P-UT. Then also $\left(\eta^{(n)}\right)_{n \in \mathbb{N}}$ is P-UT (cf. 
[15, Eq. VI.6.3, p. 377]).

From [15, Thm. VI.6.22, p. 383] it now follows that

$$
\left(\xi^{(n)}, \eta^{(n)}, \int_{0}^{\cdot} e^{-\xi_{s-}^{(n)}} d \eta_{s}^{(n)}\right) \stackrel{\mathcal{L}}{\rightarrow}\left(\xi, \eta, \int_{0}^{\cdot} e^{-\xi_{s-}} d \eta_{s}\right), \quad n \rightarrow \infty,
$$

in the Skorokhod topology. Since none of the components has a discontinuity at fixed $t \geq 0$ with positive probability, this implies

$$
\left(A_{0}^{(n)}, B_{0}^{(n)}\right) \stackrel{d}{\rightarrow}\left(A_{0}, B_{0}\right), \quad n \rightarrow \infty .
$$

By assumption we have $\log \left|A_{0}^{(n)}\right|=-\xi_{1}^{(n)} \stackrel{d}{\rightarrow}-\xi_{1}=\log \left|A_{0}\right|$. Since additionally the sequence $\left(\log \left|A_{0}^{(n)}\right|\right)_{n \in \mathbb{N}}$ is uniformly integrable by (7.2) (see e.g. [7, Condition (3.18)]), this yields by [7, Thm. 3.5]

$$
E\left[\log \left|A_{0}^{(n)}\right|\right] \rightarrow E\left[\log \left|A_{0}\right|\right], \quad n \rightarrow \infty .
$$

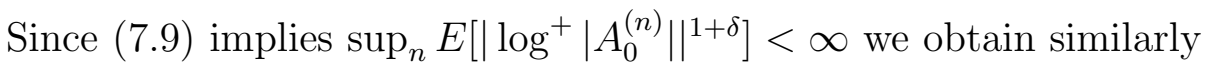

$$
E\left[\log ^{+}\left|A_{0}^{(n)}\right|\right] \rightarrow E\left[\log ^{+}\left|A_{0}\right|\right], \quad n \rightarrow \infty .
$$

Also, it is obvious that (7.3) and (7.2) yield

$$
-\infty<E\left[\log \left|A_{0}\right|\right]<0 \quad \text { and } \quad-\infty<E\left[\log \left|A_{0}^{(n)}\right|\right]<0 .
$$

Finally, observe that (7.9) implies

$$
\sup _{n \in \mathbb{N}} E\left[\log ^{+} \sup _{0<s \leq 1}\left|e^{-\xi_{s}^{(n)}}\right|\right]^{1+\delta}<\infty
$$

which, together with (7.1) and (7.10), yields by Lemma 7.4

$$
\sup _{n \in \mathbb{N}} E\left[\log ^{+}\left|B_{0}^{(n)}\right|\right]^{1+\delta}<\infty .
$$

Again, this gives $E\left[\log ^{+}\left|B_{0}\right|\right]^{1+\delta}<\infty$ and

$$
E\left[\log ^{+}\left|B_{0}^{(n)}\right|\right] \rightarrow E\left[\log ^{+}\left|B_{0}\right|\right]<\infty, \quad n \rightarrow \infty .
$$

Now, by Proposition 7.2 we obtain the stated result from (7.12), (7.13), (77.14), (17.15) and (7.16).

From the above theorem we immediately obtain the following corollary on injectivity of $\Phi_{\xi}$ and $\tilde{\Phi}_{\eta}$. Observe that the conditions in part $(i)$ have been violated in Example 7.1 .

Corollary 7.5. (i) Let $\left(\xi_{t}\right)_{t \geq 0}$ be a Lévy process such that $E\left[\xi_{1}\right]>0$ and $E\left[\left|\xi_{1}\right|^{1+\delta}\right]<\infty$ for some $\delta>0$. Let $\left(\eta^{(n)}\right)_{n \in \mathbb{N}}$ be a sequence of Lévy processes such that $\eta_{1}^{(n)} \stackrel{d}{\rightarrow} \eta_{1}$ as $n \rightarrow \infty, \mathcal{L}\left(\eta_{1}^{(n)}\right) \in D_{\xi}, \mathcal{L}\left(\eta_{1}\right) \in D_{\xi}$ and

$$
\sup _{n \in \mathbb{N}} \int_{|x|>1}\left(\log ^{+}|x|\right)^{1+\delta} \nu_{\eta^{(n)}}(d x)<\infty .
$$

Then $\Phi_{\xi}\left(\mathcal{L}\left(\eta_{1}^{(n)}\right)\right) \stackrel{w}{\rightarrow} \Phi_{\xi}\left(\mathcal{L}\left(\eta_{1}\right)\right)$ as $n \rightarrow \infty$. 
(ii) Let $\left(\eta_{t}\right)_{t \geq 0}$ be a Lévy process such that $E\left[\log ^{+}\left|\eta_{1}\right|^{1+\delta}\right]<\infty$ for some $\delta>0$. Let $\left(\xi^{(n)}\right)_{n \in \mathbb{N}}$ be a sequence of Lévy processes such that $\xi_{1}^{(n)} \stackrel{d}{\rightarrow} \xi_{1}$ as $n \rightarrow \infty, \mathcal{L}\left(\xi_{1}^{(n)}\right) \in$ $\tilde{D}_{\eta}, \mathcal{L}\left(\xi_{1}\right) \in \tilde{D}_{\eta}, E\left[\xi_{1}^{(n)}\right]>0, E\left[\xi_{1}\right]>0$ and

$$
\sup _{n \in \mathbb{N}} E\left[\left|\xi_{1}^{(n)}\right|^{1+\delta}\right]<\infty
$$

Then $\tilde{\Phi}_{\eta}\left(\mathcal{L}\left(\xi_{1}^{(n)}\right)\right) \stackrel{w}{\rightarrow} \tilde{\Phi}_{\eta}\left(\mathcal{L}\left(\xi_{1}\right)\right)$ as $n \rightarrow \infty$.

\section{Acknowledgement}

The authors would like to thank Makoto Maejima for fruitful discussions which initiated the investigations of this paper.

\section{References}

[1] G. Alsmeyer, A. Iksanov and U. Rösler (2009) On distributional properties of perpetuities. J. Theoret. Probab. 22, 666-682.

[2] D. Applebaum (2004) Lévy Processes and Stochastic Calculus. Cambridge University Press, Cambridge.

[3] A. Behme (2011) Distributional properties of solutions of $d V_{t}=V_{t-} d U_{t}+d L_{t}$ with Lévy noise. Adv. Appl. Prob. 43, 688-711.

[4] A. Behme, A. Lindner and R. Maller (2011) Stationary solutions of the stochastic differential equation $d V_{t}=V_{t-} d U_{t}+d L_{t}$ with Lévy noise. Stoch. Process. Appl. 121, 91-108.

[5] A. Behme, M. Maejima, M. Matsui and N. Sakuma (2012) Distributions of exponential integrals of independent increment processes related to generalized gamma convolutions. Bernoulli, 18, 1172-1187.

[6] J. Bertoin, A. Lindner and R. Maller (2008) On continuity properties of the law of integrals of Lévy processes. In: C. Donati-Martin, M. Émery, A. Rouault, C. Stricker (eds.): Séminaire de Probabilités XLI, Lecture Notes in Mathematics 1934, 137-159, Springer, Berlin.

[7] P. Billingsley (1999) Convergence of Probability Measures. 2nd edition. Wiley Series in Probability and Statistics, New York.

[8] A. Brandt (1986) The stochastic equation $Y_{n+1}=A_{n} Y_{n}+B_{n}$ with stationary coefficients. Adv. Appl. Prob. 18, 211-220.

[9] P. Carmona (1996) Some complements to "On the distribution and asymptotic results for exponential functionals of Lévy processes". Unpublished. 
[10] P. Carmona, F. Petit and M. Yor (1997) On the distribution and asymptotic results for exponential functionals of Lévy processes. In M. Yor (ed.): Exponential Functionals and Principal Values Related to Brownian Motion, Bibl. Rev. Mat. Iberoamericana, Rev. Mat. Iberoamericana, Madrid, 73-130.

[11] P. Carmona, F. Petit and M. Yor (2001) Exponential functionals of Lévy Processes. In O.E. Barndorff-Nielsen, T. Mikosch, S. Resnick (eds.): Lévy Processes, Birkhäuser Boston, Boston, MA, 41-55.

[12] K.B. Erickson and R.A. Maller (2005) Generalised Ornstein-Uhlenbeck processes and the convergence of Lévy integrals. In M. Emery, M. Ledoux, M. Yor (eds.): Séminaire de Probabilités XXXVIII, Lecture Notes in Mathematics 1857, 70-94, Springer, Berlin.

[13] S. Ethier and T. Kurtz (1986) Markov Processes: Characterization and Convergence, Wiley Series in Probability and Mathematical Statistics, New York.

[14] A.I. Il'inskii (1975) On the zeros and argument of characteristic functions. Theory Probab. Appl. 29, 410-415.

[15] J. Jacod and A.N. Shiryaev (2003) Limit Theorems for Stochastic Processes. 2nd edition. Springer, New-York.

[16] Z.J. Jurek and J.D. Mason (1993) Operator-limit Distributions in Probability Theory. Wiley, New York.

[17] O. Kallenberg (2001) Foundations of Modern Probability. 2nd edition, Springer, Berlin.

[18] R.L. Karandikar (1991) Multiplicative decomposition of nonsingular matrix valued semimartingales. In J. Azéma, M. Yor, P. Meyer (eds.): Séminaire de Probabilités XXV, Lecture Notes in Math. 1485, 262-269, Springer, Berlin.

[19] T. Kato (1976) Perturbation Theory for Linear Operators. 2nd edition. Springer, Berlin.

[20] T. Kawata (1972) Fourier Analysis in Probability Theory. Academic Press, New York and London.

[21] V. Kolokoltsov (2011) Markov Processes, Semigroups and Generators. De Gruyter Studies in Mathematics 38, Berlin.

[22] H. Kondo; M. Maejima and K. Sato (2006) Some properties of exponential integrals of Lévy processes and examples. Electr. Comm. Probab. 11, 291-303.

[23] A. Kuznetsov, J.C. Pardo and M. Savov (2012) Distributional properties of exponential functionals of Lévy processes. Electron. J. Probab. 17, 1-35.

[24] A. Kyprianou (2006) Introductory Lectures on Fluctuations of Lévy Processes with Applications. Springer, Berlin. 
[25] P. Lévy (1961) Quelques problèmes non résolus de la théorie des fonctions caractéristiques. Ann. Mat. Pura Appl. 53(4), 315-331.

[26] T. Liggett (2010) Continuous Time Markov Processes. An Introduction. AMS Graduate Studies in Mathematics 113. Providence, RI.

[27] A. Lindner and R. Maller (2005) Lévy integrals and the stationarity of generalised Ornstein-Uhlenbeck processes. Stoch. Process. Appl. 115, 1701-1722

[28] A. Lindner and K. Sato (2009) Continuity properties and infinite divisibility of stationary distributions of some generalised Ornstein-Uhlenbeck processes. Ann. Probab. 37, 250-274.

[29] M. Loève (1945) Nouvelles classes de lois limites. Bulletin de la S.M.F. 73, 107-126.

[30] E. Lucasz (1970) Characteristic Functions. 2nd edition. Griffin, London.

[31] K. Maulik and B. Zwart (2006) Tail asymptotics for exponential functionals of Lévy processes. Stoch. Process. Appl. 116, 156-177.

[32] P. Medvegyev (2007) Stochastic Integration Theory. Oxford University Press, Oxford.

[33] T. Nilsen and J. Paulsen (1996) On the distribution of a randomly discounted compound Poisson process. Stoch. Process. Appl. 61, 305-310.

[34] J. Paulsen (2003) Risk theory in a stochastic economic environment. Stoch. Process. Appl. 46, 327-361.

[35] P.E. Protter (2005) Stochastic Integration and Differential Equations. 2nd edition, Version 2.1, Springer, Berlin.

[36] K. Sato (1999) Lévy Processes and Infinitely Divisible Distributions. Cambridge University Press, Cambridge.

[37] K. Sato (2007) Transformations of infinitely divisible distributions via improper stochastic integrals. ALEA 3, 67-110.

[38] K. Sato and M. Yamazato (1984) Operator-selfdecomposable distributions as limit distributions of processes of Ornstein-Uhlenbeck type. Stoch. Process. Appl. 17, 73100 .

[39] R. L. Schilling and A. Schnurr (2010) The symbol associated with the solution of a stochastic differential equation. Electron. J. Probab. 15, 1369-1393.

[40] F. W. Steutel and K. van Harn (2003) Infinite Divisibility of Probability Distributions on the Real Line. Marcel Dekker Inc, New York.

[41] M. Yor (2001) Exponential Functionals of Brownian Motion and Related Processes. Springer Finance, Berlin. 